SUPPORTING INFORMATION

JULY 17, 2019

\title{
Multiple Bonds in Uranium-Transition Metal Complexes
}

\author{
Prachi Sharma, ${ }^{\dagger, \ddagger}$ Dale Pahls, ${ }^{\dagger, \ddagger}$ Bianca L. Ramirez, ${ }^{\dagger}$ Connie C. Lu, ${ }^{\dagger}$ and Laura \\ Gagliardit,‡,*
}

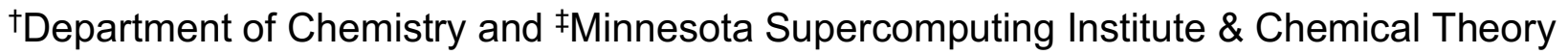
Center, University of Minnesota, Minneapolis, Minnesota 55455-0431, United States

* Corresponding authors

\section{Table of contents}

Table S1. Detailed CASSCF orbital analysis of UTM-2 species. S-2 Table S2. CASPT2/SO-RASSI excitation energies with respect to the S-3 ground state in eV for UMn-2.

Figure S1. UMn-2 orbitals predicted by CASSCF $(10,13)$ for the S-4 singlet state along with the occupation number of the orbitals.

Figure S2. UMn-2 orbitals predicted by CASSCF $(12,13)$ for the S-4 singlet state along with the occupation number of the orbitals.

Figure S3. Infrared spectra for UMn-2 complex calculated at DFT S-5 level of theory.

Table S3. Absolute ground state CASSCF energies for UTM-2 S-5 complexes in atomic units.

Table S4. Absolute excited state CASSCF and MS-CASPT2 S-6 energies for UMn-2 complex in atomic units.

Figure S4. A comparison between the $\mathrm{MO}$ diagrams of $\mathrm{UMn}(\mathrm{CO})_{3}{ }^{-}$and $\quad \mathrm{S}-7$ UMn-2 complexes.

Table S5. Cartesian coordinates ( $\AA$ ) for UTM-2 complexes for $\quad$ S-7 different spin states. 
Table S1. Detailed CASSCF orbital analysis of UTM-2 species.

\begin{tabular}{|c|c|c|c|c|c|c|c|}
\hline Complex & Orbital & \%TM \%U & $\begin{array}{c}\text { total } \\
\text { electrons }\end{array}$ & $\begin{array}{l}\text { Electron } \\
\text { TM }\end{array}$ & $\begin{array}{c}\text { Electron } \\
U\end{array}$ & $\mathrm{EBO}^{\mathrm{a}}$ & $\begin{array}{c}\text { Mayer } \\
\text { BO }^{\text {b }}\end{array}$ \\
\hline \multirow{4}{*}{$\mathrm{UCr}-2$} & $\sigma$ & 48.551 .5 & 1.78 & 0.86 & 0.92 & \multirow{4}{*}{3.3} & \multirow{4}{*}{3.3} \\
\hline & $\pi$ & 63.236 .8 & 1.91 & 1.21 & 0.70 & & \\
\hline & $\pi$ & 66.533 .6 & 1.91 & 1.27 & 0.64 & & \\
\hline & $\delta$ & 52.247 .8 & 1.67 & 0.87 & 0.80 & & \\
\hline \multirow{5}{*}{ UMn-2 } & $\sigma$ & 60.239 .8 & 1.85 & 1.11 & 0.74 & \multirow{5}{*}{4.3} & \multirow{5}{*}{3.6} \\
\hline & $\pi$ & 68.731 .3 & 1.91 & 1.31 & 0.60 & & \\
\hline & $\pi$ & 69.730 .3 & 1.91 & 1.33 & 0.58 & & \\
\hline & $\delta$ & 75.724 .3 & 1.81 & 1.42 & 0.43 & & \\
\hline & $\delta$ & 71.328 .7 & 1.77 & 1.26 & 0.51 & & \\
\hline \multirow{5}{*}{ UFe-2 } & $\sigma$ & 65.334 .7 & 1.82 & 1.19 & 0.63 & \multirow{5}{*}{2.7} & \multirow{5}{*}{2.9} \\
\hline & $\pi$ & 77.722 .3 & 1.91 & 1.48 & 0.43 & & \\
\hline & $\pi$ & 75.424 .6 & 1.91 & 1.44 & 0.47 & & \\
\hline & $3 d(F e)-5 f / 6 d(U)$ & $95.7 \quad 4.3$ & 1.92 & 1.84 & 0.08 & & \\
\hline & $3 d(F e)-5 f / 6 d(U)$ & 97.22 .8 & 1.93 & 1.88 & 0.05 & & \\
\hline \multirow{4}{*}{ UCo-2 } & $3 \mathrm{~d} / 4 \mathrm{p}(\mathrm{Co})-6 \mathrm{~d}(\mathrm{U})$ & 88.210 .3 & 1.93 & 1.70 & 0.20 & \multirow{4}{*}{1.9} & \multirow{4}{*}{1.6} \\
\hline & $\begin{array}{c}3 d / 4 p(C o)- \\
5 f / 6 d(U)\end{array}$ & 88.310 .0 & 1.93 & 1.70 & 0.19 & & \\
\hline & $3 d(C o)-5 f / 6 d(U)$ & $91.8 \quad 8.2$ & 1.94 & 1.78 & 0.16 & & \\
\hline & $3 d(C o)-5 f / 6 d(U)$ & $95.9 \quad 4.2$ & 1.94 & 1.86 & 0.08 & & \\
\hline \multirow{2}{*}{ UNi-2 } & $\begin{array}{l}3 d / 4 s / 4 p(N i)- \\
5 f / 6 d(U)\end{array}$ & 34.557 .7 & 1.01 & 0.35 & 0.58 & \multirow{2}{*}{0.5} & \multirow{2}{*}{0.7} \\
\hline & $\begin{array}{c}3 \mathrm{~d} / 4 \mathrm{~s}(\mathrm{Ni})- \\
7 \mathrm{~s} / 6 \mathrm{~d}(\mathrm{U})\end{array}$ & 88.611 .4 & 1.95 & 1.72 & 0.22 & & \\
\hline
\end{tabular}

a Only natural MO's with overlap percentage higher than 10\% from either metal are considered as bonding MO's

${ }^{\mathrm{b}}$ Calculated at PBE-D3 level of theory 
Table S2. CASPT2/SO-RASSI excitation energies with respect to the ground state in eV for UMn-2.

\begin{tabular}{|c|c|}
\hline State & $E(e V)$ \\
\hline 1 & 0.00 \\
\hline 2 & 1.31 \\
\hline 3 & 1.32 \\
\hline 4 & 1.39 \\
\hline 5 & 1.52 \\
\hline 6 & 1.52 \\
\hline 7 & 1.53 \\
\hline 8 & 1.60 \\
\hline 9 & 1.77 \\
\hline 10 & 1.90 \\
\hline 11 & 1.91 \\
\hline 12 & 1.94 \\
\hline 13 & 2.05 \\
\hline 14 & 2.07 \\
\hline 15 & 2.12 \\
\hline 16 & 2.21 \\
\hline
\end{tabular}

SO-RASSI: Spin-orbit restricted active space state interaction 


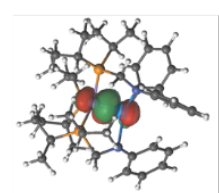

$\pi_{u}(5 f / 6 d(U)-3 d(M n)$ 1.91

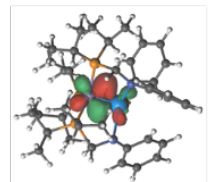

$\pi_{u}(5 f / 6 d(U)-3 d(M n)$

1.91

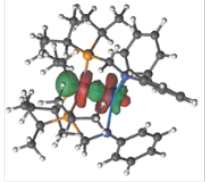

$\sigma_{g}(5 f(U)-3 d(M n)$

1.82

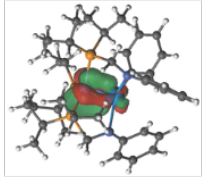

$\delta_{g}(5 f(U)-3 d(M n)$

1.80

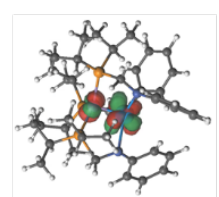

$\delta_{u}(5 f(U)-3 d(M n)$

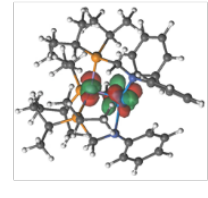

$\delta_{u}(5 f(U)-3 d(M n)$

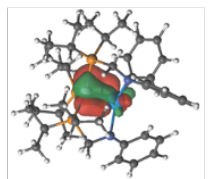

$\delta_{g}(5 f(U)-3 d(M n)$

1.76

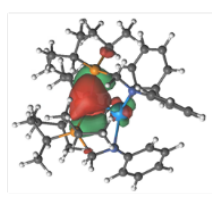

$5 f / 6 d(U)-3 d(M n)$

0.03

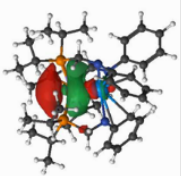

$5 f / 6 d(U)-3 d(M n)$ 0.02

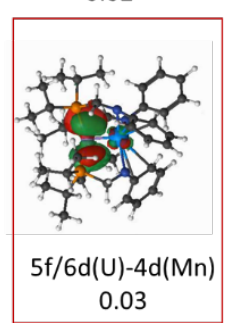

Figure S1. UMn-2 orbitals predicted by CASSCF $(10,13)$ for the singlet state along with the occupation number of the orbitals. The highlighted orbital is the extra orbital as compared to $(10,12)$ active space. The occupation numbers do not change significantly on the expansion of active space from $(10,12)$ to $(10,13)$.

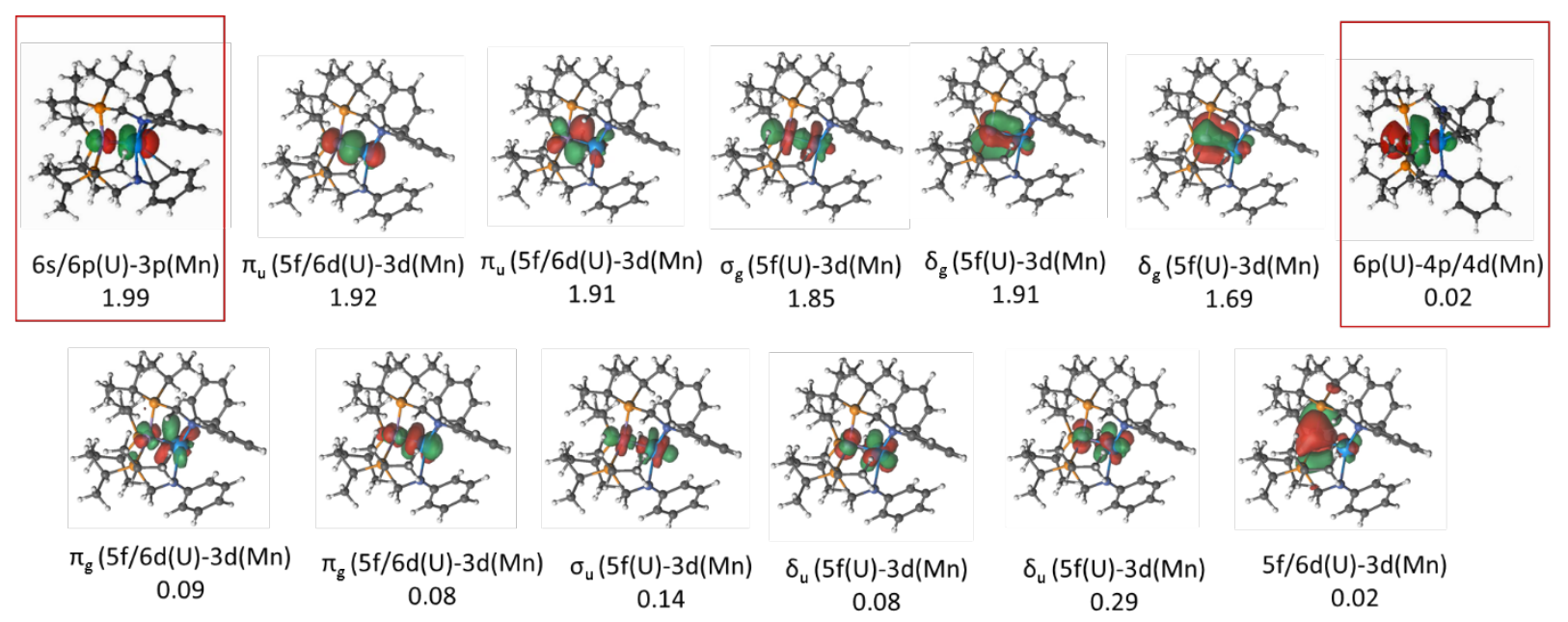

Figure S2. UMn-2 orbitals predicted by CASSCF $(12,13)$ for the singlet state along with the occupation number of the orbitals. The highlighted orbitals are different/extra orbital as compared to $(10,12)$ active space. The highlighted orbitals consist of a doubly occupied orbital with contributions from core like $6 s$ and $6 p$ orbitals of $U$ and $3 p$ orbitals of $\mathrm{Mn}$, and its correlating orbital. 


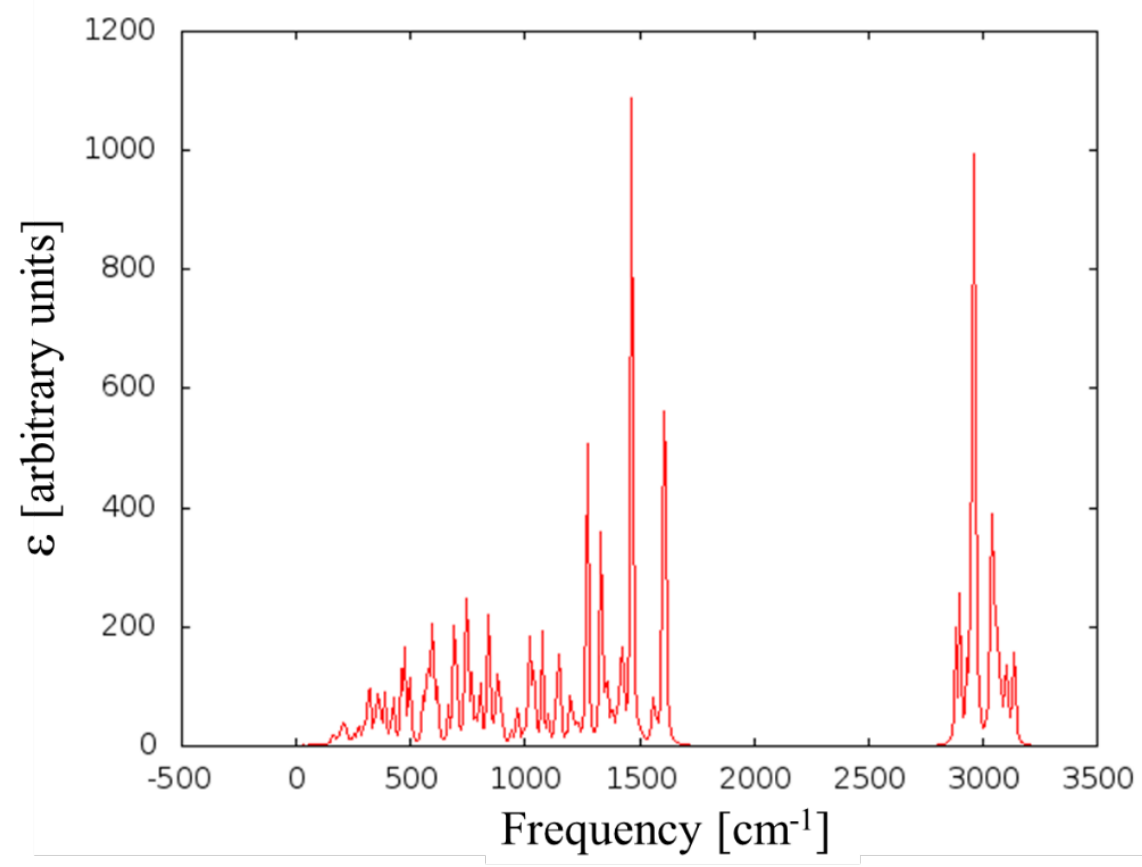

Figure S3. Infrared spectra for UMn-2 complex calculated at DFT level of theory.

Table S3. Absolute ground state CASSCF energies for UTM-2 complexes in atomic units.

\begin{tabular}{|cccc|}
\hline UTM-2 & Active - space & Spin & CASSCF \\
\hline UCr-2 & $(9,12)$ & 2 & -31700.300744 \\
UMn-2 & $(10,12)$ & 1 & -31808.005604 \\
UFe-2 & $(11,12)$ & 2 & -31922.069345 \\
UCo-2 & $(12,12)$ & 3 & -32042.676958 \\
UNi-2 & $(13,12)$ & 4 & -32169.689777 \\
\hline
\end{tabular}


Table S4. Absolute excited state CASSCF and MS-CASPT2 energies for UMn-2 complex in atomic units.

\begin{tabular}{|ccc|}
\hline State & CASSCF & MS-CASPT2 \\
\hline$S_{0}$ & -31807.997814 & -31812.214044 \\
$S_{1}$ & -31807.952763 & -31812.149387 \\
$S_{2}$ & -31807.947309 & -31812.146314 \\
$S_{3}$ & -31807.944047 & -31812.141250 \\
$T_{1}$ & -31807.957504 & -31812.159628 \\
$T_{2}$ & -31807.951700 & -31812.153265 \\
$T_{3}$ & -31807.949063 & -31812.150074 \\
$T_{4}$ & -31807.945417 & -31812.147258 \\
\hline
\end{tabular}

\section{Exploring other possible multiply bonded UTM complexes}

Recently, Chi et al. reported the synthesis and characterization of the $\mathrm{UFe}(\mathrm{CO})_{3}{ }^{-}$complex with a three-folded bonding between $\mathrm{U}$ and $\mathrm{Fe}$, which is similar in nature to the bonds observed for UFe-2 (Section 3.3). In UFe $(\mathrm{CO})_{3}{ }^{-}$, the $U$ atom is in the formal +1 oxidation state while $\mathrm{Fe}$ is in the -2 oxidation state. We extended this study to the $\mathrm{UMn}(\mathrm{CO})_{3}{ }^{-}$system to explore the bonding between $\mathrm{U}$ and $\mathrm{Mn}$ in the presence of a new ligand (CO). Our calculations show that $\mathrm{UMn}(\mathrm{CO})_{3}{ }^{-}$also contains five-fold bonds. We cannot directly compare the effective bond orders of $\mathrm{UMn}(\mathrm{CO})_{3}{ }^{-}$and $\mathrm{UMn}-2$ as the active-spaces are different owing to the different natures of the ligands. Figure S4 shows a comparison between the MO diagrams in $\mathrm{UMn}(\mathrm{CO})_{3}{ }^{-}$and UMn-2. 


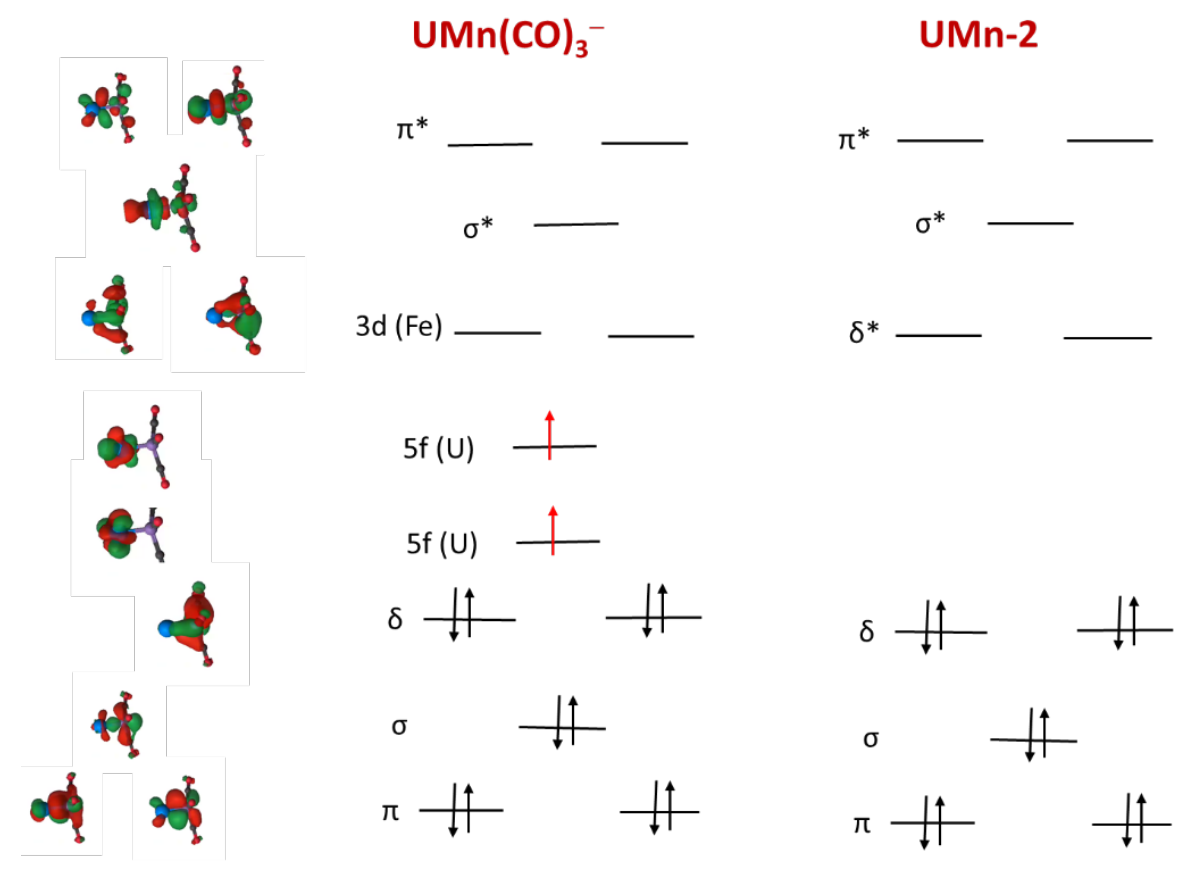

Figure S4. A comparison between the $\mathrm{MO}$ diagrams of $\mathrm{UMn}(\mathrm{CO})_{3}{ }^{-}$and $\mathrm{UMn}-2$ complexes. Electrons localized at $\mathrm{U}$ are shown in red.

Table S5. Cartesian coordinates $(\AA)$ for UTM-2 complexes for different spin states. The spin refers to $S(S+1)$.

UCr-2 (Spin 2)

$$
\begin{array}{cccc}
\text { P } & 2.522751 & 0.429685 & -1.288897 \\
\text { P } & 1.411032 & 0.516046 & 2.386914 \\
\text { P } & -0.114258 & -2.616064 & 0.377881 \\
\text { N } & 0.130346 & 1.329250 & -2.335110 \\
\text { N } & -2.216773 & -1.006659 & 0.577981 \\
\text { N } & -0.336047 & 2.256853 & 1.457595 \\
\text { C } & -4.180686 & 0.364095 & 0.162374 \\
\text { C } & 4.019203 & 1.582895 & -1.121385 \\
\text { H } & 4.270999 & 1.860067 & -2.169848
\end{array}
$$



C $\quad-1.966339 \quad 3.109725 \quad-0.030252$
C $\quad-0.122148-0.511124 \quad-4.025371$
H $\quad 0.932858 \quad-0.497192-4.332065$
C $\quad-2.347874-1.528315-4.202429$
H $\quad-3.005723 \quad-2.297358 \quad-4.632480$
C $\quad 1.550284 \quad 1.474854-2.538232$
H $\quad 1.829112 \quad 2.530332 \quad-2.348134$
$\begin{array}{llll}H & 1.884604 & 1.246642 & -3.575117\end{array}$
$\begin{array}{llll}\text { C } & -2.353316 & 3.945546 & -1.097637\end{array}$
H $\quad-3.334668 \quad 3.789685-1.573864$
C $\quad-3.488000 \quad-0.596985 \quad 0.957510$
C $\quad-0.603134 \quad 0.444768-3.072610$
C $\quad-4.158145 \quad-1.057870 \quad 2.129810$
H $\quad-3.686658 \quad-1.820320 \quad 2.765308$
C $\quad-0.985929-1.461460-4.573973$
H $\quad-0.587893 \quad-2.185024 \quad-5.303647$
$\begin{array}{llll}\text { C } & 0.100819 & 4.400698 & 0.231484\end{array}$
H $\quad 1.044882 \quad 4.6108890 .753782$
C $\quad-1.521305 \quad 4.987871 \quad-1.516922$
H $\quad-1.818327 \quad 5.644427 \quad-2.347373$
$\begin{array}{llll}\text { C } & 0.869866 & 2.298175 & 2.266222\end{array}$
H $\quad 0.681457 \quad 2.750471 \quad 3.268930$
H $1.703611 \quad 2.864602 \quad 1.796915$
C $\quad-5.416848 \quad 0.892260 \quad 0.552929$
H $\quad-5.914001 \quad 1.634360-0.092173$
C $\quad \begin{array}{llll}-5.401811 & -0.533331 & 2.504397\end{array}$
H $\quad-5.883176 \quad-0.905073 \quad 3.423334$
$\begin{array}{llll}\text { C } & 5.247812 & 0.924492 & -0.482928\end{array}$ 


$$
\begin{array}{lrrr}
\mathrm{H} & 6.047081 & 1.678140 & -0.318279 \\
\mathrm{H} & 5.679056 & 0.124036 & -1.112750 \\
\mathrm{H} & 5.003852 & 0.478721 & 0.501810 \\
\mathrm{C} & -0.311603 & 5.218249 & -0.821739 \\
\mathrm{C} & -0.699360 & 3.282363 & 0.630721 \\
\mathrm{C} & -2.853307 & -0.576593 & -3.308526 \\
\mathrm{H} & -3.921697 & -0.575442 & -3.041659 \\
\mathrm{C} & -2.007299 & 0.411778 & -2.771871 \\
\mathrm{C} & 0.506220 & 0.014516 & 3.967999 \\
\mathrm{H} & 0.895270 & 0.685879 & 4.763476 \\
\mathrm{C} & -1.751668 & -2.258830 & 1.156571 \\
\mathrm{H} & -2.482103 & -3.090294 & 1.013158 \\
\mathrm{H} & -1.558153 & -2.199225 & 2.254966 \\
\mathrm{C} & 3.198434 & -0.975111 & -2.371744 \\
\mathrm{H} & 2.245723 & -1.380998 & -2.776739 \\
\mathrm{C} & -1.004503 & 0.250634 & 3.842766 \\
\mathrm{H} & -1.260697 & 1.320873 & 3.734247 \\
\mathrm{H} & -1.528823 & -0.138621 & 4.740779 \\
\mathrm{H} & -1.426685 & -0.261327 & 2.955762 \\
\mathrm{C} & 3.834043 & -2.085381 & -1.523724 \\
\mathrm{H} & 4.816761 & -1.787749 & -1.108448 \\
\mathrm{H} & 3.997191 & -2.999785 & -2.132896 \\
\mathrm{H} & 3.179856 & -2.355167 & -0.669780 \\
\mathrm{C} & -6.039111 & 0.456205 & 1.735548 \\
\mathrm{H} & -7.015313 & 0.861995 & 2.039415 \\
\mathrm{H} & 4.085658 & -0.566301 & -3.552907 \\
\hline & 3.622433 & 0.218124 & -4.185232 \\
\hline
\end{array}
$$



H $\quad 5.073589-0.187364 \quad-3.220962$
C $\quad 0.463044 \quad-4.098483 \quad 1.385209$
$\begin{array}{llll}H & 0.289787 & -3.717627 & 2.416677\end{array}$
C $\quad 3.204179 \quad 0.666818 \quad 2.955036$
H $3.601144 \quad 1.370503 \quad 2.191487$
C $\quad 3.438387 \quad 1.287035 \quad 4.336884$
H $4.520615 \quad 1.4817294 .496372$
H $\quad 3.109807 \quad 0.611819 \quad 5.153533$
H $\quad 2.906755 \quad 2.252104 \quad 4.462461$
C $\quad-0.592596-3.206301-1.351773$
H $\quad-0.684704 \quad-2.233515 \quad-1.881315$
$\begin{array}{llll}\text { C } & 3.645118 & 2.865812 & -0.369175\end{array}$
H $3.504204 \quad 2.663717 \quad 0.710986$
H $\quad 2.710215 \quad 3.333134-0.736196$
H $\quad 4.456547 \quad 3.617993 \quad-0.461370$
C $-1.941695-3.919006-1.497569$
H $\quad-2.780448 \quad-3.277606 \quad-1.164518$
H $\quad-1.986871 \quad-4.876757 \quad-0.943548$
H $\quad-2.118443 \quad-4.144062 \quad-2.570409$
C $\quad 1.974811-4.314553 \quad 1.238866$
H $\quad 2.336194 \quad-5.067901 \quad 1.970482$
H $2.527939 \quad-3.369908 \quad 1.416876$
H $\quad 2.246068 \quad-4.678810 \quad 0.227621$
C $\quad-0.334314 \quad-5.400293 \quad 1.235509$
H $\quad 0.002767 \quad-6.144869 \quad 1.988213$
H $\quad-0.189170 \quad-5.860645 \quad 0.237873$
H $\quad-1.421814 \quad-5.248978 \quad 1.383855$
$\begin{array}{llll}\text { C } & 0.548989 & -3.970474 & -2.033035\end{array}$ 


$$
\begin{array}{lrrr}
\mathrm{H} & 0.343989 & -4.063260 & -3.120112 \\
\mathrm{H} & 0.665882 & -4.996435 & -1.628946 \\
\mathrm{H} & 1.519430 & -3.450594 & -1.919667 \\
\mathrm{C} & 3.952962 & -0.660315 & 2.771768 \\
\mathrm{H} & 5.049966 & -0.512616 & 2.863382 \\
\mathrm{H} & 3.659225 & -1.411908 & 3.531723 \\
\mathrm{H} & 3.741516 & -1.096866 & 1.773435 \\
\mathrm{C} & 0.821011 & -1.435734 & 4.346413 \\
\mathrm{H} & 1.885959 & -1.587492 & 4.609341 \\
\mathrm{H} & 0.212819 & -1.757431 & 5.217824 \\
\mathrm{H} & 0.590188 & -2.117091 & 3.501846 \\
\mathrm{H} & 0.326964 & 6.065789 & -1.119442 \\
\mathrm{U} & -0.627723 & 0.605532 & -0.201414 \\
\mathrm{Cr} & 0.968485 & -0.474109 & 0.282712 \\
\mathrm{H} & -2.436406 & 1.291566 & -2.257447 \\
\mathrm{H} & -3.753702 & 0.647323 & -0.810415 \\
\mathrm{H} & -2.708237 & 2.436290 & 0.436230
\end{array}
$$

UCr-2 (Spin 4)

$$
\begin{array}{lrrr}
P & -2.454781 & 0.244669 & -1.519426 \\
P & -1.456242 & -1.586228 & 1.989419 \\
P & -0.098733 & 2.220361 & 1.334481 \\
N & 0.022375 & -0.091608 & -2.642330 \\
N & 2.097272 & 0.769748 & 1.053854 \\
N & 0.352119 & -2.700936 & 0.430939 \\
C & 4.131104 & -0.288164 & 0.269370 \\
C & -3.862294 & -0.962817 & -1.859124 \\
\text { H } & -4.107079 & -0.817130 & -2.935113
\end{array}
$$




$$
\begin{array}{lrrr}
\mathrm{C} & 2.020420 & -2.752907 & -1.244798 \\
\mathrm{C} & 0.395263 & 2.264252 & -3.422780 \\
\mathrm{H} & -0.658082 & 2.431366 & -3.689206 \\
\mathrm{C} & 2.662225 & 3.166357 & -3.180507 \\
\mathrm{H} & 3.360442 & 4.012110 & -3.261165 \\
\mathrm{C} & -1.366583 & -0.114965 & -3.023455 \\
\mathrm{H} & -1.633022 & -1.137478 & -3.361708 \\
\mathrm{H} & -1.619162 & 0.566907 & -3.866072 \\
\mathrm{C} & 2.452554 & -3.053721 & -2.560087 \\
\mathrm{H} & 3.433440 & -2.683566 & -2.899575 \\
\mathrm{C} & 3.369390 & 0.295046 & 1.326832 \\
\mathrm{C} & 0.818817 & 0.982859 & -2.944731 \\
\mathrm{C} & 3.976871 & 0.313664 & 2.618118 \\
\mathrm{H} & 3.451575 & 0.779088 & 3.463986 \\
\mathrm{C} & 1.306697 & 3.318682 & -3.539285 \\
\mathrm{H} & 0.948361 & 4.294887 & -3.904675 \\
\mathrm{C} & 0.007337 & -4.121881 & -1.614117 \\
\mathrm{H} & -0.926292 & -4.576311 & -1.251858 \\
\mathrm{C} & 1.670138 & -3.843815 & -3.401282 \\
\mathrm{H} & 2.003602 & -4.073204 & -4.423780 \\
\mathrm{C} & -0.843673 & -3.127697 & 1.127606 \\
\mathrm{H} & -0.633816 & -3.945212 & 1.857947 \\
\mathrm{H} & -1.658089 & -3.490432 & 0.460559 \\
\mathrm{C} & 5.373170 & -0.889880 & 0.500524 \\
\mathrm{H} & 5.924668 & -1.330766 & -0.345396 \\
\mathrm{C} & 5.227713 & -0.279414 & 2.835127 \\
\mathrm{C} & 5.660642 & -0.257110 & 3.848400 \\
\hline & -5.120811 & -0.697879 & -1.024725
\end{array}
$$




$$
\begin{array}{lrrr}
\mathrm{H} & -5.864257 & -1.508021 & -1.181705 \\
\mathrm{H} & -5.615107 & 0.255837 & -1.289019 \\
\mathrm{H} & -4.886931 & -0.664638 & 0.058517 \\
\mathrm{C} & 0.462765 & -4.397869 & -2.903039 \\
\mathrm{C} & 0.751074 & -3.249513 & -0.757125 \\
\mathrm{C} & 3.111031 & 1.905377 & -2.758940 \\
\mathrm{H} & 4.174352 & 1.745940 & -2.520143 \\
\mathrm{C} & 2.218771 & 0.825131 & -2.677975 \\
\mathrm{C} & -0.566726 & -1.724057 & 3.639545 \\
\mathrm{H} & -0.920630 & -2.681210 & 4.080538 \\
\mathrm{C} & 1.512703 & 1.654148 & 2.044529 \\
\mathrm{H} & 2.178178 & 2.511958 & 2.302735 \\
\mathrm{H} & 1.269082 & 1.151448 & 3.013380 \\
\mathrm{C} & -3.181071 & 1.933113 & -1.949744 \\
\mathrm{H} & -2.244985 & 2.514834 & -2.099974 \\
\mathrm{C} & 0.953268 & -1.816483 & 3.452134 \\
\mathrm{H} & 1.258691 & -2.716088 & 2.886485 \\
\mathrm{H} & 1.455021 & -1.842893 & 4.441962 \\
\mathrm{H} & 1.353801 & -0.948374 & 2.892648 \\
\mathrm{C} & -3.923157 & 2.560427 & -0.762838 \\
\mathrm{H} & -4.914140 & 2.094604 & -0.596609 \\
\mathrm{H} & -4.095255 & 3.643889 & -0.935773 \\
\mathrm{H} & -3.341782 & 2.447379 & 0.172776 \\
\mathrm{C} & 5.933139 & -0.901805 & 1.790532 \\
\mathrm{H} & 6.913890 & -1.365348 & 1.972889 \\
\mathrm{H} & -4.000928 & 1.996771 & -3.243616 \\
- & -3.463087 & 1.570086 & -4.113981 \\
\hline & -4.242763 & 3.051681 & -3.493182
\end{array}
$$




$$
\begin{array}{lrrr}
\text { H } & -4.966065 & 1.458746 & -3.148073 \\
\text { C } & -0.835574 & 3.117364 & 2.818665 \\
\text { H } & -0.708765 & 2.332521 & 3.598174 \\
\text { C } & -3.249299 & -2.012455 & 2.382536 \\
\text { H } & -3.604938 & -2.344343 & 1.381996 \\
\text { C } & -3.464897 & -3.165877 & 3.368976 \\
\text { H } & -4.538808 & -3.447160 & 3.407197 \\
\text { H } & -3.169106 & -2.884561 & 4.400553 \\
\text { H } & -2.894476 & -4.074685 & 3.089331 \\
\mathrm{C} & 0.431519 & 3.485731 & 0.040393 \\
\text { H } & 0.570234 & 2.830534 & -0.845522 \\
\mathrm{C} & -3.375217 & -2.403181 & -1.665425 \\
\mathrm{H} & -3.179965 & -2.604202 & -0.593825 \\
\mathrm{H} & -2.437035 & -2.627070 & -2.210160 \\
\mathrm{H} & -4.146773 & -3.124312 & -2.007597 \\
\mathrm{C} & 1.768660 & 4.195751 & 0.278999 \\
\mathrm{H} & 2.604996 & 3.474109 & 0.351640 \\
\mathrm{H} & 1.763861 & 4.830189 & 1.187288 \\
\mathrm{H} & 1.988319 & 4.852296 & -0.588907 \\
\mathrm{C} & -2.343170 & 3.352325 & 2.670580 \\
\mathrm{H} & -2.778873 & 3.703136 & 3.630012 \\
\mathrm{H} & -2.862658 & 2.416355 & 2.380880 \\
\mathrm{H} & -2.572136 & 4.119697 & 1.904433 \\
\mathrm{C} & -0.091812 & 4.370520 & 3.299679 \\
\mathrm{H} & -0.513116 & 4.717932 & 4.267291 \\
\mathrm{H} & -0.192293 & 5.209649 & 2.582842 \\
\hline & -0.989414 & 4.187572 & 3.456310 \\
\hline
\end{array}
$$




$$
\begin{array}{lrrr}
\text { H } & -0.449209 & 5.002140 & -1.246438 \\
H & -0.835455 & 5.235191 & 0.483625 \\
H & -1.663970 & 3.957544 & -0.453334 \\
\mathrm{C} & -4.056717 & -0.760315 & 2.751195 \\
\mathrm{H} & -5.146191 & -0.973527 & 2.727634 \\
\mathrm{H} & -3.815433 & -0.394399 & 3.769447 \\
\mathrm{H} & -3.846100 & 0.067780 & 2.042622 \\
\mathrm{C} & -0.954542 & -0.574677 & 4.576417 \\
\mathrm{H} & -2.029979 & -0.581410 & 4.839768 \\
\mathrm{H} & -0.377914 & -0.633864 & 5.523026 \\
\mathrm{H} & -0.734297 & 0.406803 & 4.108543 \\
\mathrm{H} & -0.131425 & -5.064278 & -3.548857 \\
\mathrm{U} & 0.697518 & -0.526751 & -0.404673 \\
\mathrm{Cr} & -1.044046 & 0.198027 & 0.439974 \\
\mathrm{H} & 2.596537 & -0.203187 & -2.532417 \\
\mathrm{H} & 3.754646 & -0.199896 & -0.761131 \\
\mathrm{H} & 2.751338 & -2.391914 & -0.495673
\end{array}
$$

$$
\begin{aligned}
& \text { UCr-2 (Spin 6) } \\
& \begin{array}{cccc}
\text { P } & 2.530227 & -0.378934 & -1.406172 \\
\text { P } & 1.386000 & 1.796798 & 1.855975 \\
\text { P } & 0.036416 & -2.051059 & 1.540635 \\
\text { N } & 0.085703 & -0.144619 & -2.641868 \\
\text { N } & -2.129502 & -0.605154 & 1.071371 \\
\text { N } & -0.358249 & 2.718869 & 0.149286 \\
\text { C } & -4.196515 & 0.259457 & 0.123462 \\
\text { C } & 3.965805 & 0.780035 & -1.809936 \\
\text { H } & 4.236869 & 0.537052 & -2.861697
\end{array}
\end{aligned}
$$



C $\quad-2.046130 \quad 2.624932 \quad-1.516856$
C $\quad-0.178327 \quad-2.571303 \quad-3.229386$
H $\quad 0.881654 \quad-2.713004 \quad-3.482994$
C $\quad-2.408165 \quad-3.548434 \quad-2.936685$
H $\quad-3.070632 \quad-4.425859 \quad-2.962190$
C $1.488404-0.115745 \quad-2.962311$
H $1.7512680 .894499-3.336406$
H $1.790855-0.831230 \quad-3.759991$
C $\quad-2.482966 \quad 2.807147 \quad-2.853111$
H $\quad-3.472870 \quad 2.423023 \quad-3.147757$
C $\quad-3.435849 \quad-0.166568 \quad 1.252778$
$\begin{array}{llll}\text { C } & -0.659526 & -1.276819 & -2.853387\end{array}$
C $\quad-4.079867 \quad-0.088145 \quad 2.523143$
H $\quad-3.551883 \quad-0.423262 \quad 3.427045$
C $\quad-1.044967 \quad-3.668863 \quad-3.275691$
H $\quad-0.643779-4.651945-3.571288$
C $\quad-0.010862 \quad 3.912842-2.035592$
H $\quad 0.934584 \quad 4.381742 \quad-1.725135$
$\begin{array}{llll}\text { C } & -1.693828 & 3.495202 & -3.773198\end{array}$
H $\quad-2.033434 \quad 3.629790 \quad-4.810577$
$\begin{array}{llll}\text { C } & 0.823427 & 3.231474 & 0.807795\end{array}$
H $\quad 0.607957 \quad 4.136604 \quad 1.426075$
H $\quad 1.656415 \quad 3.499660 \quad 0.118742$
C $\quad-5.481896 \quad 0.794419 \quad 0.260510$
H $\quad-6.031539 \quad 1.111894-0.640145$
C $\quad \begin{array}{llll}-5.373098 & 0.438438 & 2.648086\end{array}$
H $\quad \begin{array}{llll}-5.833146 & 0.493508 & 3.648054\end{array}$
$\begin{array}{llll}\text { C } & 5.197404 & 0.570750 & -0.921298\end{array}$ 


$$
\begin{array}{lrrr}
\mathrm{H} & 5.963535 & 1.345850 & -1.135671 \\
\mathrm{H} & 5.675581 & -0.414171 & -1.078396 \\
\mathrm{H} & 4.937479 & 0.650091 & 0.153893 \\
\mathrm{C} & -0.469204 & 4.069648 & -3.343358 \\
\mathrm{C} & -0.768099 & 3.153249 & -1.088195 \\
\mathrm{C} & -2.911528 & -2.280385 & -2.605021 \\
\mathrm{H} & -3.980765 & -2.148150 & -2.376648 \\
\mathrm{C} & -2.065642 & -1.161794 & -2.598857 \\
\mathrm{C} & 0.437737 & 2.115490 & 3.448505 \\
\mathrm{H} & 0.788957 & 3.110976 & 3.797187 \\
\mathrm{C} & -1.570010 & -1.384055 & 2.161661 \\
\mathrm{H} & -2.251836 & -2.199721 & 2.502529 \\
\mathrm{H} & -1.328876 & -0.784192 & 3.073929 \\
\mathrm{C} & 3.251645 & -2.103288 & -1.679349 \\
\mathrm{H} & 2.313535 & -2.685676 & -1.810358 \\
\mathrm{C} & -1.074797 & 2.202301 & 3.205869 \\
\mathrm{H} & -1.349640 & 3.037830 & 2.536096 \\
\mathrm{H} & -1.603241 & 2.343450 & 4.171981 \\
\mathrm{H} & -1.471695 & 1.282541 & 2.733328 \\
\mathrm{C} & 3.944434 & -2.638183 & -0.419250 \\
\mathrm{H} & 4.929231 & -2.162006 & -0.246138 \\
\mathrm{H} & 4.118093 & -3.732028 & -0.500569 \\
\mathrm{H} & 3.324072 & -2.459748 & 0.481157 \\
\mathrm{C} & -6.084376 & 0.898253 & 1.527369 \\
\mathrm{H} & -7.098462 & 1.310763 & 1.635294 \\
\mathrm{H} & 4.111401 & -2.284261 & -2.935208 \\
\mathrm{H} & 3.606634 & -1.925341 & -3.854704 \\
& 4.345386 & -3.359083 & -3.089250
\end{array}
$$



H $\quad 5.080740 \quad-1.751301 \quad-2.854516$
C $\quad 0.724105 \quad-2.822605 \quad 3.119087$
H $\quad 0.584406 \quad-1.973634 \quad 3.825628$
C $\quad 3.167624 \quad 2.253753 \quad 2.281102$
H $3.564992 \quad 2.501253 \quad 1.272180$
C $\quad 3.359736 \quad 3.481801 \quad 3.177708$
H $4.433996 \quad 3.759917 \quad 3.230556$
H $\quad 3.025327 \quad 3.288922 \quad 4.217749$
H $\quad 2.806738 \quad 4.367000 \quad 2.803497$
C $\quad-0.503122 \quad-3.419094 \quad 0.361413$
$\begin{array}{llll}\text { H } & -0.636597 & -2.846077 & -0.580604\end{array}$
$\begin{array}{llll}\text { C } & 3.505647 & 2.241314 & -1.756271\end{array}$
H $3.292326 \quad 2.543109-0.712036$
H $2.585596 \quad 2.435378-2.341833$
H $4.300330 \quad 2.912475 \quad-2.144133$
$\begin{array}{llll}\text { C } & -1.843346 & -4.100460 & 0.659306\end{array}$
H $\quad-2.676715 \quad-3.3718120 .670927$
H $\quad-1.841751 \quad-4.6554581 .617839$
H $\quad-2.065528-4.826863 \quad-0.150108$
C $\quad 2.232829-3.080491 \quad 3.028420$
H $\quad 2.641187 \quad-3.366964 \quad 4.020735$
H $\quad 2.773477 \quad-2.172500 \quad 2.690428$
H $\quad 2.473937 \quad-3.900736 \quad 2.323116$
C $\quad-0.040838 \quad-4.025806 \quad 3.685118$
H $\quad 0.352119 \quad-4.291214 \quad 4.689929$
H $\quad 0.071799 \quad-4.9234023 .045009$
H $\quad-1.124075 \quad-3.823220 \quad 3.796124$
$\begin{array}{llll}\text { C } & 0.621665 & -4.429547 & 0.105988\end{array}$ 


$$
\begin{array}{lrrr}
\mathrm{H} & 0.374960 & -5.047862 & -0.781905 \\
\mathrm{H} & 0.767397 & -5.118950 & 0.962147 \\
\mathrm{H} & 1.589138 & -3.931320 & -0.094285 \\
\mathrm{C} & 3.947586 & 1.029355 & 2.780857 \\
\mathrm{H} & 5.038283 & 1.236615 & 2.803795 \\
\mathrm{H} & 3.647170 & 0.732270 & 3.805454 \\
\mathrm{H} & 3.780182 & 0.151885 & 2.120406 \\
\mathrm{C} & 0.782322 & 1.069910 & 4.515334 \\
\mathrm{H} & 1.849048 & 1.094838 & 4.811637 \\
\mathrm{H} & 0.178045 & 1.236485 & 5.431388 \\
\mathrm{H} & 0.562577 & 0.045292 & 4.150589 \\
\mathrm{H} & 0.132308 & 4.656474 & -4.055979 \\
\mathrm{U} & -0.702771 & 0.504282 & -0.500515 \\
\mathrm{Cr} & 1.195042 & -0.200583 & 0.579014 \\
\mathrm{H} & -2.489094 & -0.142700 & -2.513416 \\
\mathrm{H} & -3.771770 & 0.117348 & -0.881465 \\
\mathrm{H} & -2.775076 & 2.341678 & -0.734630
\end{array}
$$

UCr-2 (Spin 8)

$$
\begin{array}{lrrr}
\mathrm{P} & 2.672514 & 0.192618 & -1.426375 \\
\mathrm{P} & 1.184477 & 1.330764 & 2.240380 \\
\mathrm{P} & 0.317408 & -2.456431 & 0.947390 \\
\mathrm{~N} & 0.109495 & 0.410650 & -2.448633 \\
\mathrm{~N} & -2.017405 & -1.161148 & 0.838446 \\
\mathrm{~N} & -0.719487 & 2.585732 & 0.890689 \\
\mathrm{C} & -4.124666 & -0.147117 & 0.210586 \\
\mathrm{C} & 3.860732 & 1.648008 & -1.548489 \\
\mathrm{H} & 4.128340 & 1.708534 & -2.626859
\end{array}
$$




$$
\begin{aligned}
& \text { C } \quad-2.377385 \quad 2.894380-0.784746 \\
& \text { C } \quad-0.185092-1.739447 \quad-3.709411 \\
& \text { H } \quad 0.891535 \quad-1.865714 \quad-3.897070 \\
& \text { C } \quad-2.457482-2.647850-3.854655 \\
& \text { H } \quad-3.139555-3.452568 \quad-4.165285 \\
& \begin{array}{llll}
\text { C } & 1.484973 & 0.602108 & -2.836357
\end{array} \\
& \text { H } \quad 1.654977 \quad 1.672584 \quad-3.081295 \\
& \text { H } \quad 1.773558 \quad 0.028666 \quad-3.744790 \\
& \text { C } \quad-2.808224 \quad 3.415693 \quad-2.026688 \\
& \text { H } \quad-3.778177 \quad 3.089735 \quad-2.435782 \\
& \begin{array}{llll}
\text { C } & -3.338468 & -0.879090 & 1.151691
\end{array} \\
& \text { C } \quad-0.658943-0.572787-3.029440 \\
& \begin{array}{llll}
\text { C } & -3.974755 & -1.235111 & 2.378039
\end{array} \\
& \text { H } \quad-3.429822 \quad-1.824173 \quad 3.129142 \\
& \text { C } \quad-1.073350-2.740464 \quad-4.114124 \\
& \text { H } \quad-0.675934 \quad-3.628314 \quad-4.632535 \\
& \begin{array}{llll}
\text { C } & -0.389304 & 4.332632 & -0.892655
\end{array} \\
& \text { H } \quad 0.538042 \quad 4.727563 \quad-0.452512 \\
& \text { C } \quad-2.041143 \quad 4.363845 \quad-2.706841 \\
& \text { H } \quad-2.379834 \quad 4.771187 \quad-3.670678 \\
& \text { C } \quad 0.446573 \quad 2.943956 \quad 1.661800 \\
& \text { H } \quad 0.201547 \quad 3.603070 \quad 2.531182 \\
& \text { H } \quad 1.239384 \quad 3.45964111 .074260 \\
& \text { C } \quad-5.426518 \quad 0.272385 \quad 0.505605
\end{aligned}
$$

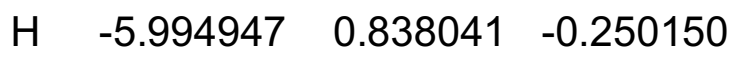

$$
\begin{aligned}
& \text { C } \quad-5.285449-0.822045 \quad 2.656253 \\
& \text { H } \quad-5.741590 \quad-1.107117 \quad 3.618259 \\
& \begin{array}{llll}
\text { C } & 5.142691 & 1.449915 & -0.731134
\end{array}
\end{aligned}
$$




$\begin{array}{lrrr}\mathrm{H} & 5.774575 & 2.362126 & -0.777062 \\ \mathrm{H} & 5.757059 & 0.605580 & -1.097130 \\ \mathrm{H} & 4.913743 & 1.258285 & 0.336987 \\ \mathrm{C} & -0.844483 & 4.835399 & -2.112358 \\ \mathrm{C} & -1.126233 & 3.317913 & -0.203586 \\ \mathrm{C} & -2.950155 & -1.493707 & -3.228789 \\ \mathrm{H} & -4.031690 & -1.374392 & -3.057544 \\ \mathrm{C} & -2.077284 & -0.459369 & -2.853907 \\ \mathrm{C} & 0.282124 & 1.076154 & 3.872041 \\ \mathrm{H} & 0.534783 & 1.967994 & 4.485535 \\ \mathrm{C} & -1.363399 & -2.160480 & 1.660464 \\ \mathrm{H} & -1.948134 & -3.109869 & 1.725507 \\ \mathrm{H} & -1.192391 & -1.834026 & 2.717453 \\ \mathrm{C} & 3.639752 & -1.254237 & -2.175677 \\ \mathrm{H} & 2.794830 & -1.913228 & -2.478933 \\ \mathrm{C} & -1.239294 & 1.048903 & 3.676246 \\ \mathrm{H} & -1.629355 & 1.990881 & 3.248007 \\ \mathrm{H} & -1.742382 & 0.874499 & 4.650352 \\ \mathrm{H} & -1.551978 & 0.239597 & 2.987339 \\ \mathrm{C} & 4.445477 & -2.012230 & -1.112117 \\ \mathrm{H} & 5.344816 & -1.450943 & -0.789178 \\ \mathrm{H} & 4.791350 & -2.993178 & -1.501301 \\ \mathrm{H} & 3.832039 & -2.204272 & -0.206889 \\ \mathrm{C} & -6.020891 & -0.052875 & 1.738541 \\ \mathrm{H} & -7.047965 & 0.266709 & 1.968787 \\ \mathrm{C} & 4.472175 & -0.938417 & -3.423374 \\ \mathrm{H} & 3.886571 & -0.409681 & -4.202510 \\ & 4.856787 & -1.874404 & -3.881700\end{array}$



H $\quad 5.354864-0.311155 \quad-3.182924$
C $\quad \begin{array}{lll}1.056605 & -3.593234 & 2.261209\end{array}$
$\begin{array}{llll}H & 0.821088 & -3.016624 & 3.183981\end{array}$
$\begin{array}{llll}\text { C } & 2.924621 & 1.867774 & 2.758055\end{array}$
H $\quad 3.248898 \quad 2.421185 \quad 1.849744$
$\begin{array}{llll}\text { C } & 2.997090 & 2.827469 & 3.951222\end{array}$
H $\quad 4.030422 \quad 3.217849 \quad 4.068439$
H $\quad 2.732793 \quad 2.322482 \quad 4.902872$
H $\quad 2.325063 \quad 3.701005 \quad 3.832862$
$\begin{array}{llll}\text { C } & -0.042544 & -3.441560 & -0.612735\end{array}$
H $\quad-0.226246-2.619349-1.336201$
$\begin{array}{llll}\text { C } & 3.153780 & 2.948325 & -1.151795\end{array}$
H $2.9045692 .940213 \quad-0.072470$
H $\quad 2.203735 \quad 3.112852 \quad-1.696862$
H $\quad 3.811465 \quad 3.822338$-1.342974
C $\quad-1.301501 \quad-4.314689-0.611990$
H $\quad-2.209940 \quad-3.717387 \quad-0.403072$
H $\quad-1.247241 \quad-5.149770 \quad 0.113845$
H $\quad-1.435470 \quad-4.754523 \quad-1.622493$
$\begin{array}{llll}\text { C } & 2.585145 & -3.664107 & 2.153431\end{array}$
H $\quad 3.016868 \quad-4.192064 \quad 3.029889$
H $\quad 3.032197 \quad-2.647797 \quad 2.116785$
H $\quad 2.913243 \quad-4.207063 \quad 1.244626$
$\begin{array}{llll}\text { C } & 0.415342 & -4.979486 & 2.402388\end{array}$
H $\quad 0.818253 \quad-5.499956 \quad 3.297483$
H $\quad 0.632586-5.622970 \quad 1.526644$
H $\quad-0.684884 \quad-4.925479 \quad 2.517593$
$\begin{array}{llll}\text { C } & 1.197974 & -4.194294 & -1.108495\end{array}$ 


$\begin{array}{lrrr}\mathrm{H} & 1.046388 & -4.517345 & -2.159599 \\ \mathrm{H} & 1.404325 & -5.104187 & -0.508869 \\ \mathrm{H} & 2.104708 & -3.558529 & -1.080857 \\ \mathrm{C} & 3.870630 & 0.669177 & 2.917672 \\ \mathrm{H} & 4.923999 & 1.009078 & 3.008878 \\ \mathrm{H} & 3.638029 & 0.067749 & 3.818842 \\ \mathrm{H} & 3.813819 & -0.013075 & 2.039422 \\ \mathrm{C} & 0.785254 & -0.180235 & 4.591560 \\ \mathrm{H} & 1.850055 & -0.107483 & 4.887209 \\ \mathrm{H} & 0.195086 & -0.364208 & 5.513463 \\ \mathrm{H} & 0.681147 & -1.074216 & 3.942128 \\ \mathrm{H} & -0.259592 & 5.618817 & -2.620650 \\ \mathrm{U} & -0.882932 & 0.599402 & -0.327565 \\ \mathrm{Cr} & 1.512119 & -0.420291 & 0.564111 \\ \mathrm{H} & -2.482925 & 0.523361 & -2.542945 \\ \mathrm{H} & -3.712672 & 0.027431 & -0.796658 \\ \mathrm{H} & -3.086351 & 2.339879 & -0.143475\end{array}$

UMn-2 (Spin 1)

$$
\begin{array}{lrrr}
P & -2.457427 & -0.160725 & -1.422782 \\
P & -1.361234 & -1.014806 & 2.276884 \\
P & -0.117091 & 2.416384 & 0.729110 \\
N & -0.016829 & -0.820638 & -2.549730 \\
N & 2.138310 & 0.963729 & 0.737781 \\
N & 0.456008 & -2.486891 & 1.047221 \\
C & 4.213578 & -0.196126 & 0.218357 \\
C & -3.853983 & -1.443778 & -1.453325 \\
H & -4.109767 & -1.557581 & -2.530760
\end{array}
$$




$$
\begin{array}{lrrr}
\mathrm{C} & 2.098604 & -2.993021 & -0.578574 \\
\mathrm{C} & 0.289252 & 1.306569 & -3.846814 \\
\mathrm{H} & -0.758723 & 1.361694 & -4.172789 \\
\mathrm{C} & 2.517636 & 2.327545 & -3.759354 \\
\mathrm{H} & 3.187564 & 3.161217 & -4.014933 \\
\mathrm{C} & -1.429494 & -0.905240 & -2.830558 \\
\mathrm{H} & -1.715050 & -1.972868 & -2.904820 \\
\mathrm{H} & -1.720572 & -0.438402 & -3.796853 \\
\mathrm{C} & 2.509785 & -3.622126 & -1.771641 \\
\mathrm{H} & 3.473806 & -3.339956 & -2.224393 \\
\mathrm{C} & 3.436316 & 0.622090 & 1.091391 \\
\mathrm{C} & 0.742145 & 0.186158 & -3.078315 \\
\mathrm{C} & 4.057575 & 1.019821 & 2.312669 \\
\mathrm{H} & 3.519314 & 1.672092 & 3.013971 \\
\mathrm{C} & 1.169239 & 2.337396 & -4.182255 \\
\mathrm{H} & 0.793550 & 3.187548 & -4.774186 \\
\mathrm{C} & 0.103010 & -4.415097 & -0.516523 \\
\mathrm{H} & -0.822415 & -4.755078 & -0.030483 \\
\mathrm{C} & 1.725028 & -4.624491 & -2.348779 \\
\mathrm{H} & 2.041773 & -5.120788 & -3.277372 \\
\mathrm{C} & -0.732106 & -2.721693 & 1.848172 \\
\mathrm{H} & -0.502980 & -3.321648 & 2.760504 \\
\mathrm{H} & -1.543872 & -3.247367 & 1.300144 \\
\mathrm{C} & 5.489949 & -0.648187 & 0.571249 \\
\mathrm{H} & 6.051863 & -1.279640 & -0.135483 \\
\mathrm{C} & 5.341085 & 0.571354 & 2.651234 \\
\mathrm{C} & 5.782481 & -0.891571 & 3.608823 \\
& -5.115399 & -1.013723 & -0.695786
\end{array}
$$




$$
\begin{array}{lrrr}
\text { H } & -5.840655 & -1.853462 & -0.643039 \\
\text { H } & -5.634235 & -0.164062 & -1.177842 \\
\text { H } & -4.879148 & -0.713429 & 0.344391 \\
\mathrm{C} & 0.539040 & -5.027521 & -1.692129 \\
\mathrm{C} & 0.853603 & -3.338685 & 0.055910 \\
\mathrm{C} & 2.993844 & 1.226253 & -3.038574 \\
\mathrm{H} & 4.050960 & 1.173739 & -2.735024 \\
\mathrm{C} & 2.132616 & 0.157959 & -2.726750 \\
\mathrm{C} & -0.475894 & -0.775163 & 3.926904 \\
\mathrm{H} & -0.807183 & -1.622795 & 4.565458 \\
\mathrm{C} & 1.546246 & 2.073809 & 1.473922 \\
\mathrm{H} & 2.203068 & 2.974595 & 1.470904 \\
\mathrm{H} & 1.350594 & 1.831415 & 2.546323 \\
\mathrm{C} & -3.264956 & 1.330969 & -2.262372 \\
\mathrm{H} & -2.352095 & 1.880700 & -2.581393 \\
\mathrm{C} & 1.045075 & -0.875278 & 3.752400 \\
\mathrm{H} & 1.370748 & -1.876014 & 3.412727 \\
\mathrm{H} & 1.556521 & -0.654669 & 4.712634 \\
\mathrm{H} & 1.417269 & -0.153962 & 2.998640 \\
\mathrm{C} & -3.992223 & 2.215707 & -1.242289 \\
\mathrm{H} & -4.962813 & 1.783322 & -0.930074 \\
\mathrm{H} & -4.201934 & 3.220652 & -1.666236 \\
\mathrm{H} & -3.377940 & 2.349979 & -0.330442 \\
\mathrm{C} & 6.067629 & -0.276380 & 1.797913 \\
\mathrm{H} & 7.074601 & -0.623100 & 2.073119 \\
\mathrm{H} & -4.120997 & 1.053576 & -3.502928 \\
-3.595593 & 0.432243 & -4.256323 \\
\hline
\end{array}
$$




$$
\begin{array}{lrrr}
\mathrm{H} & -5.069037 & 0.538123 & -3.247125 \\
\mathrm{C} & -0.815604 & 3.601975 & 2.015060 \\
\mathrm{H} & -0.638528 & 3.009587 & 2.940035 \\
\mathrm{C} & -3.144471 & -1.339147 & 2.785699 \\
\mathrm{H} & -3.508754 & -1.913162 & 1.906076 \\
\mathrm{C} & -3.358495 & -2.199169 & 4.036034 \\
\mathrm{H} & -4.432496 & -2.460875 & 4.146713 \\
\mathrm{H} & -3.060522 & -1.664919 & 4.961324 \\
\mathrm{H} & -2.788683 & -3.149703 & 3.996787 \\
\mathrm{C} & 0.344016 & 3.411645 & -0.810853 \\
\mathrm{H} & 0.504607 & 2.584767 & -1.534047 \\
\mathrm{C} & -3.352765 & -2.796546 & -0.936738 \\
\mathrm{H} & -3.193117 & -2.761243 & 0.158563 \\
\mathrm{H} & -2.391827 & -3.111801 & -1.389688 \\
\mathrm{H} & -4.099605 & -3.592469 & -1.140487 \\
\mathrm{C} & 1.641181 & 4.228281 & -0.776159 \\
\mathrm{H} & 2.523142 & 3.590762 & -0.572999 \\
\mathrm{H} & 1.617677 & 5.047953 & -0.031385 \\
\mathrm{H} & 1.806307 & 4.689317 & -1.773113 \\
\mathrm{C} & -2.333931 & 3.767437 & 1.880963 \\
\mathrm{H} & -2.745126 & 4.314372 & 2.755809 \\
\mathrm{H} & -2.837853 & 2.781847 & 1.828320 \\
\mathrm{H} & -2.611907 & 4.339844 & 0.973622 \\
\mathrm{C} & -0.096104 & 4.946368 & 2.181701 \\
\mathrm{H} & -0.491277 & 5.484325 & 3.070030 \\
\mathrm{H} & -0.251879 & 5.608136 & 1.306615 \\
\hline & -0.837830 & 4.232894 & -1.338296
\end{array}
$$




$$
\begin{array}{cccc}
\mathrm{H} & -0.638934 & 4.559309 & -2.380845 \\
\mathrm{H} & -1.012925 & 5.146547 & -0.734519 \\
\mathrm{H} & -1.776412 & 3.648467 & -1.344190 \\
\mathrm{C} & -3.945377 & -0.030077 & 2.824569 \\
\mathrm{H} & -5.034966 & -0.234389 & 2.890168 \\
\mathrm{H} & -3.675218 & 0.598754 & 3.696331 \\
\mathrm{H} & -3.759685 & 0.571732 & 1.911143 \\
\mathrm{C} & -0.878305 & 0.537561 & 4.604471 \\
\mathrm{H} & -1.951327 & 0.568706 & 4.876248 \\
\mathrm{H} & -0.293739 & 0.692919 & 5.535371 \\
\mathrm{H} & -0.680587 & 1.400681 & 3.936567 \\
\mathrm{H} & -0.061289 & -5.846666 & -2.120131 \\
\mathrm{U} & 0.639149 & -0.556882 & -0.299114 \\
\mathrm{Mn} & -0.989150 & 0.265763 & 0.354825 \\
\mathrm{H} & 2.546192 & -0.801647 & -2.364923 \\
\mathrm{H} & 3.816464 & -0.428836 & -0.779713 \\
\mathrm{H} & 2.813810 & -2.370976 & -0.010219
\end{array}
$$

UMn-2 (Spin 3)

$$
\begin{array}{lrrr}
\mathrm{P} & -2.450434 & -0.477582 & -1.314586 \\
\mathrm{P} & -1.476931 & -0.465779 & 2.334479 \\
\mathrm{P} & 0.035152 & 2.558471 & 0.328599 \\
\mathrm{~N} & -0.005817 & -1.293179 & -2.289933 \\
\mathrm{~N} & 2.187198 & 1.050170 & 0.627310 \\
\mathrm{~N} & 0.298949 & -2.241474 & 1.562038 \\
\mathrm{C} & 4.191941 & -0.273640 & 0.293107 \\
\mathrm{C} & -3.928288 & -1.655235 & -1.199126 \\
\mathrm{H} & -4.116802 & -1.941775 & -2.258435
\end{array}
$$




$$
\begin{array}{lrrr}
\mathrm{C} & 1.961365 & -3.031883 & 0.083489 \\
\mathrm{C} & 0.241117 & 0.475846 & -4.053471 \\
\mathrm{H} & -0.821280 & 0.464212 & -4.334320 \\
\mathrm{C} & 2.468077 & 1.466214 & -4.321578 \\
\mathrm{H} & 3.125278 & 2.207890 & -4.798432 \\
\mathrm{C} & -1.410036 & -1.497975 & -2.526060 \\
\mathrm{H} & -1.655927 & -2.559082 & -2.319899 \\
\mathrm{H} & -1.732603 & -1.299679 & -3.572985 \\
\mathrm{C} & 2.388424 & -3.869761 & -0.976022 \\
\mathrm{H} & 3.378851 & -3.698485 & -1.427576 \\
\mathrm{C} & 3.460556 & 0.693864 & 1.045102 \\
\mathrm{C} & 0.732151 & -0.438541 & -3.067527 \\
\mathrm{C} & 4.097824 & 1.215448 & 2.209834 \\
\mathrm{H} & 3.594166 & 1.985083 & 2.811585 \\
\mathrm{C} & 1.101218 & 1.395499 & -4.662286 \\
\mathrm{H} & 0.693118 & 2.088580 & -5.415849 \\
\mathrm{C} & -0.087035 & -4.382448 & 0.300476 \\
\mathrm{H} & -1.030024 & -4.622341 & 0.812746 \\
\mathrm{C} & 1.587116 & -4.922525 & -1.414934 \\
\mathrm{H} & 1.916296 & -5.569882 & -2.240667 \\
\mathrm{C} & -0.953330 & -2.257996 & 2.293111 \\
\mathrm{H} & -0.833265 & -2.679178 & 3.319170 \\
\mathrm{H} & -1.759831 & -2.831852 & 1.787815 \\
\mathrm{C} & 5.436768 & -0.750202 & 0.719310 \\
\mathrm{H} & 5.965000 & -1.499961 & 0.108680 \\
\mathrm{C} & 5.351220 & 0.741645 & 2.619646 \\
\mathrm{C} & 5.808147 & 1.159159 & 3.531475 \\
\hline & -5.207572 & -1.026730 & -0.633807
\end{array}
$$




$$
\begin{array}{lrrr}
\mathrm{H} & -5.995278 & -1.801199 & -0.518268 \\
\mathrm{H} & -5.620735 & -0.236376 & -1.287704 \\
\mathrm{H} & -5.036559 & -0.577506 & 0.364234 \\
\mathrm{C} & 0.364229 & -5.186587 & -0.745586 \\
\mathrm{C} & 0.680007 & -3.249682 & 0.721176 \\
\mathrm{C} & 2.981441 & 0.546373 & -3.393998 \\
\mathrm{H} & 4.054106 & 0.548356 & -3.144202 \\
\mathrm{C} & 2.141031 & -0.410225 & -2.805472 \\
\mathrm{C} & -0.650644 & 0.061134 & 3.946491 \\
\mathrm{H} & -1.074173 & -0.609965 & 4.724981 \\
\mathrm{C} & 1.656130 & 2.288038 & 1.169789 \\
\mathrm{H} & 2.350935 & 3.150538 & 1.033070 \\
\mathrm{H} & 1.427704 & 2.242100 & 2.262927 \\
\mathrm{C} & -3.104299 & 0.917286 & -2.413127 \\
\mathrm{H} & -2.142573 & 1.344840 & -2.771899 \\
\mathrm{C} & 0.866379 & -0.166004 & 3.897527 \\
\mathrm{H} & 1.133409 & -1.234396 & 3.800171 \\
\mathrm{H} & 1.340159 & 0.226613 & 4.821609 \\
\mathrm{H} & 1.332429 & 0.348865 & 3.034230 \\
\mathrm{C} & -3.803433 & 2.006452 & -1.589317 \\
\mathrm{H} & -4.794506 & 1.682645 & -1.216247 \\
\mathrm{H} & -3.964199 & 2.919691 & -2.200738 \\
\mathrm{H} & -3.191228 & 2.287282 & -0.709615 \\
\mathrm{C} & 6.028542 & -0.253965 & 1.894499 \\
\mathrm{H} & 7.011748 & -0.619478 & 2.225784 \\
\mathrm{C} & -3.926786 & 0.497366 & -3.636662 \\
\mathrm{H} & -3.417897 & -0.271185 & -4.252507 \\
\mathrm{H} & -4.107430 & 1.374301 & -4.293909
\end{array}
$$




$$
\begin{array}{lrrr}
\mathrm{H} & -4.920970 & 0.095836 & -3.354136 \\
\mathrm{C} & -0.618766 & 4.047554 & 1.272999 \\
\mathrm{H} & -0.478893 & 3.693509 & 2.318579 \\
\mathrm{C} & -3.300389 & -0.562279 & 2.788778 \\
\mathrm{H} & -3.661873 & -1.286163 & 2.026904 \\
\mathrm{C} & -3.629089 & -1.128986 & 4.174441 \\
\mathrm{H} & -4.722442 & -1.300248 & 4.271260 \\
\mathrm{H} & -3.339468 & -0.430901 & 4.986251 \\
\mathrm{H} & -3.124492 & -2.097658 & 4.365382 \\
\mathrm{C} & 0.551673 & 3.109130 & -1.403438 \\
\mathrm{H} & 0.653499 & 2.127172 & -1.913441 \\
\mathrm{C} & -3.563984 & -2.927298 & -0.424846 \\
\mathrm{H} & -3.480947 & -2.719241 & 0.660261 \\
\mathrm{H} & -2.602508 & -3.374648 & -0.744542 \\
\mathrm{H} & -4.353700 & -3.696653 & -0.553652 \\
\mathrm{C} & 1.908673 & 3.811281 & -1.527403 \\
\mathrm{H} & 2.732685 & 3.170024 & -1.159524 \\
\mathrm{H} & 1.943548 & 4.780111 & -0.991726 \\
\mathrm{H} & 2.113760 & 4.011531 & -2.599811 \\
\mathrm{C} & -2.124960 & 4.251266 & 1.070567 \\
\mathrm{H} & -2.509805 & 5.023902 & 1.769242 \\
\mathrm{H} & -2.682922 & 3.312947 & 1.260148 \\
\mathrm{H} & -2.365048 & 4.587581 & 0.042319 \\
\mathrm{C} & 0.173623 & 5.352725 & 1.120632 \\
\mathrm{H} & -0.198153 & 6.110584 & 1.842852 \\
\mathrm{H} & 0.061502 & 5.787485 & 0.107673 \\
\mathrm{C} & 1.255862 & 5.215373 & 1.312226 \\
& -0.565413 & 3.869762 & -2.128224
\end{array}
$$




$$
\begin{array}{cccc}
H & -0.336956 & 3.926351 & -3.212849 \\
H & -0.672696 & 4.908997 & -1.756753 \\
H & -1.546998 & 3.371467 & -2.020061 \\
\mathrm{C} & -4.010262 & 0.769676 & 2.511204 \\
\mathrm{H} & -5.113031 & 0.648931 & 2.564748 \\
\mathrm{H} & -3.729693 & 1.552671 & 3.243637 \\
\mathrm{H} & -3.750126 & 1.148650 & 1.501564 \\
\mathrm{C} & -0.992961 & 1.509784 & 4.305786 \\
\mathrm{H} & -2.070402 & 1.657280 & 4.514825 \\
\mathrm{H} & -0.430964 & 1.834371 & 5.206338 \\
\mathrm{H} & -0.720873 & 2.192455 & 3.475476 \\
\mathrm{H} & -0.244695 & -6.050664 & -1.056698 \\
\mathrm{U} & 0.675950 & -0.652734 & -0.118970 \\
\mathrm{Mn} & -0.988425 & 0.443274 & 0.264964 \\
\mathrm{H} & 2.565149 & -1.243523 & -2.216932 \\
\mathrm{H} & 3.784984 & -0.604016 & -0.674445 \\
\mathrm{H} & 2.700210 & -2.403205 & 0.617039 \\
\mathrm{UMn}-2 & (\mathrm{Sp} i \mathrm{n} 5) & & \\
\mathrm{P} & -2.617950 & -0.182815 & -1.449095 \\
\mathrm{P} & -0.475720 & -2.650980 & 0.875559 \\
\mathrm{P} & -0.875323 & 1.201953 & 2.021306 \\
\mathrm{~N} & -0.428257 & 1.143852 & -2.453978 \\
\mathrm{~N} & 1.669389 & 0.949705 & 1.274447 \\
\mathrm{~N} & 0.945216 & -2.216268 & -1.346059 \\
\mathrm{C} & 3.851170 & 0.892597 & 0.221724 \\
\mathrm{C} & -3.427218 & -1.627706 & -2.346072 \\
\mathrm{H} & -3.845068 & -1.173749 & -3.272800 \\
\mathrm{C} & 2.425026 & -0.910238 & -2.684545
\end{array}
$$




\begin{tabular}{lrrr}
$\mathrm{C}$ & -1.301972 & 3.480366 & -2.207402 \\
$\mathrm{H}$ & -2.319781 & 3.216144 & -2.527645 \\
$\mathrm{C}$ & 0.248251 & 5.189915 & -1.384223 \\
$\mathrm{H}$ & 0.443635 & 6.227139 & -1.075001 \\
$\mathrm{C}$ & -1.690606 & 0.612122 & -2.897788 \\
$\mathrm{H}$ & -1.510370 & -0.209074 & -3.621219 \\
$\mathrm{H}$ & -2.342074 & 1.347224 & -3.420796 \\
$\mathrm{C}$ & 3.720870 & -0.550573 & -3.119972 \\
$\mathrm{H}$ & 3.834612 & 0.313699 & -3.793513 \\
$\mathrm{C}$ & 3.049517 & 1.017698 & 1.395899 \\
$\mathrm{C}$ & -0.275735 & 2.486196 & -2.186556 \\
$\mathrm{C}$ & 3.744980 & 1.152120 & 2.633552 \\
$\mathrm{H}$ & 3.184007 & 1.246580 & 3.573784 \\
$\mathrm{C}$ & -1.030777 & 4.800549 & -1.822899 \\
$\mathrm{H}$ & -1.844668 & 5.542948 & -1.857771 \\
$\mathrm{C}$ & 3.381760 & -2.826155 & -1.485709 \\
$\mathrm{H}$ & 3.273107 & -3.736876 & -0.878369 \\
$\mathrm{C}$ & 4.837411 & -1.292481 & -2.728941 \\
$\mathrm{H}$ & 5.845097 & -1.010971 & -3.068192 \\
$\mathrm{C}$ & 0.518785 & -3.349652 & -0.559417 \\
$\mathrm{H}$ & 1.343939 & -3.977490 & -0.156719 \\
$\mathrm{H}$ & -0.136198 & -4.032683 & -1.150069 \\
$\mathrm{C}$ & 5.246190 & 0.878608 & 0.279607 \\
$\mathrm{H}$ & 5.816258 & 0.762560 & -0.655096 \\
$\mathrm{C}$ & 5.147795 & 1.153273 & 2.678822 \\
$\mathrm{H}$ & 5.649643 & 1.261109 & 3.654227 \\
$\mathrm{C}$ & -4.569782 & -2.286300 & -1.563241 \\
\hline & -4.923047 & -3.195525 & -2.094087
\end{tabular}



H $\quad-5.442872 \quad-1.618242 \quad-1.440653$
H $\quad-4.241773 \quad-2.597318 \quad-0.550961$
C $4.649772-2.441696-1.923490$
$\begin{array}{llll}\text { C } & 2.225510 & -2.058400 & -1.827996\end{array}$
$\begin{array}{llll}\text { C } & 1.278520 & 4.232356 & -1.376107\end{array}$
H $\quad 2.296801 \quad 4.511816 \quad-1.063742$
$\begin{array}{llll}\text { C } & 1.032046 & 2.919902 & -1.794572\end{array}$
$\begin{array}{llll}\text { C } & 0.676327 & -3.048273 & 2.305151\end{array}$
H $\quad 0.828593 \quad-4.148574 \quad 2.251290$
C $\quad 0.913672 \quad 1.153013 \quad 2.492801$
H $1.201906 \quad 2.082232 \quad 3.037578$
H $\quad 1.032300 \quad 0.321605 \quad 3.234908$
C $\quad-4.019117 \quad 1.040169-1.146053$

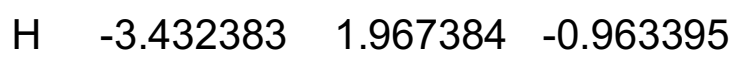
$\begin{array}{llll}\text { C } & 2.033763 & -2.359302 & 2.120668\end{array}$
H $\quad 2.554690 \quad-2.665170 \quad 1.193011$
H $\quad 2.706064 \quad-2.604106 \quad 2.968554$
H $\quad 1.932764 \quad-1.2576592 .079306$
$\begin{array}{llll}\text { C } & -4.789989 & 0.721633 & 0.141341\end{array}$
H $\quad-5.475662 \quad-0.138677 \quad 0.016117$
H $\quad-5.406888 \quad 1.590298 \quad 0.454215$
H $\quad-4.0942650 .474290 \quad 0.966769$
C $5.914583 \quad 1.013683 \quad 1.511880$
H $\quad 7.013822 \quad 1.011888 \quad 1.557417$
$\begin{array}{llll}\text { C } & -4.955264 & 1.286849 & -2.334651\end{array}$
H $\quad-4.411212 \quad 1.550986 \quad-3.263527$
H $\quad-5.644820 \quad 2.127721 \quad-2.109671$
H $\quad-5.585987 \quad 0.401455 \quad-2.554785$ 


$$
\begin{array}{llll}
\mathrm{C} & -1.679592 & 0.984161 & 3.711360 \\
\mathrm{H} & -1.147113 & 0.070467 & 4.058301 \\
\mathrm{C} & -1.893065 & -3.881305 & 1.045071 \\
\mathrm{H} & -2.263863 & -3.911822 & -0.003092 \\
\mathrm{C} & -1.486988 & -5.303786 & 1.444689 \\
\mathrm{H} & -2.353942 & -5.992486 & 1.356895 \\
\mathrm{H} & -1.140640 & -5.352951 & 2.497278 \\
\mathrm{H} & -0.678077 & -5.707797 & 0.802600 \\
\mathrm{C} & -1.126025 & 2.977285 & 1.438061 \\
\mathrm{H} & -0.882017 & 2.885158 & 0.359312 \\
\mathrm{C} & -2.374221 & -2.667419 & -2.749929 \\
\mathrm{H} & -1.987055 & -3.196913 & -1.858861 \\
\mathrm{H} & -1.494262 & -2.233245 & -3.262943 \\
\mathrm{H} & -2.823780 & -3.426364 & -3.423566 \\
\mathrm{C} & -0.175925 & 4.030223 & 2.020307 \\
\mathrm{H} & 0.881707 & 3.802661 & 1.786444 \\
\mathrm{H} & -0.282216 & 4.146692 & 3.117087 \\
\mathrm{H} & -0.399394 & 5.011053 & 1.552137 \\
\mathrm{C} & -3.165902 & 0.620618 & 3.624617 \\
\mathrm{H} & -3.548297 & 0.314188 & 4.621183 \\
\mathrm{H} & -3.325520 & -0.222491 & 2.922611 \\
\mathrm{H} & -3.786036 & 1.471886 & 3.280849 \\
\mathrm{C} & -1.405362 & 2.095368 & 4.733448 \\
\mathrm{H} & -1.774737 & 1.791605 & 5.736175 \\
\mathrm{H} & -1.925943 & 3.036532 & 4.466922 \\
\mathrm{H} & -0.325803 & 2.322397 & 4.832785 \\
\mathrm{C} & -2.592779 & 3.416377 & 1.528482 \\
\mathrm{H} & -2.743146 & 4.333559 & 0.922216
\end{array}
$$




$$
\begin{array}{llll}
H & -2.894162 & 3.651413 & 2.569319 \\
H & -3.285675 & 2.645438 & 1.144157 \\
\mathrm{C} & -3.029326 & -3.316243 & 1.907190 \\
\mathrm{H} & -3.947502 & -3.931760 & 1.801797 \\
\mathrm{H} & -2.766753 & -3.295821 & 2.983822 \\
\mathrm{H} & -3.266776 & -2.273770 & 1.606640 \\
\mathrm{C} & 0.046999 & -2.703051 & 3.658298 \\
\mathrm{H} & -0.854239 & -3.307325 & 3.877993 \\
\mathrm{H} & 0.774568 & -2.875711 & 4.478385 \\
\mathrm{H} & -0.250498 & -1.635418 & 3.693795 \\
\mathrm{H} & 5.521437 & -3.051070 & -1.636865 \\
\mathrm{U} & 0.672893 & 0.005137 & -0.699751 \\
\mathrm{Mn} & -1.180711 & -0.462150 & 0.396579 \\
\mathrm{H} & 1.879423 & 2.214467 & -1.899791 \\
\mathrm{H} & 3.368162 & 0.805527 & -0.768949 \\
\mathrm{H} & 1.544207 & -0.456284 & -3.179018
\end{array}
$$

UFe-2 (Spin 2)

$$
\begin{array}{lrrr}
P & -2.330228 & -0.017352 & -1.539308 \\
P & -1.502429 & -1.247649 & 2.062853 \\
P & -0.244984 & 2.284087 & 0.950844 \\
N & 0.198228 & -0.466548 & -2.570521 \\
N & 2.054377 & 0.915706 & 0.960718 \\
N & 0.402837 & -2.598591 & 0.848027 \\
C & 4.179357 & -0.144507 & 0.458776 \\
C & -3.689664 & -1.303327 & -1.840160 \\
H & -3.852037 & -1.279703 & -2.941235 \\
C & 2.144517 & -2.852126 & -0.727060
\end{array}
$$



C $\quad 0.561993 \quad 1.762728-3.660186$
H $\quad-0.490069 \quad 1.879515 \quad-3.956880$
$\begin{array}{llll}\text { C } & 2.815521 & 2.715012 & -3.516910\end{array}$
$\begin{array}{llll}H & 3.506839 & 3.548860 & -3.707037\end{array}$
C $-1.187532 \quad-0.549092-2.954097$
$\begin{array}{llll}\text { H } & -1.437586 & -1.605829 & -3.175719\end{array}$
$\begin{array}{llll}\text { H } & -1.434081 & 0.027910 & -3.872141\end{array}$
C $2.644264 \quad-3.330487-1.962258$
H $3.631880 \quad-2.987345 \quad-2.310032$
C $3.3329190 .560686 \quad 1.365218$
$\begin{array}{llll}C & 0.992982 & 0.567898 & -3.001293\end{array}$
$\begin{array}{llll}\text { C } & 3.865552 & 0.832461 & 2.659968\end{array}$
H $\quad 3.270560 \quad 1.398531 \quad 3.390156$
C $1.464873 \quad 2.799933 \quad-3.913804$
H $\quad 1.103122 \quad 3.710226-4.418892$
C $0.179281 \quad-4.313119-0.976298$
H $\quad-0.761462 \quad-4.732813-0.591799$
C $1.919408-4.258708-2.708916$
H $2.306010 \quad-4.628757 \quad-3.669558$
C $\quad-0.844196 \quad-2.910227 \quad 1.518135$
H $\quad-0.690673 \quad-3.595801 \quad 2.384715$
H $\quad-1.606535 \quad-3.376795 \quad 0.856836$
C $5.438993 \quad-0.616543 \quad 0.843732$
H $\quad 6.058117 \quad-1.158920 \quad 0.111250$
C $5.133923 \quad 0.3657313 .030786$
H $\quad 5.508157 \quad 0.584896 \quad 4.043894$
$\begin{array}{llll}\text { C } & -5.021868 & -0.993017 & -1.148017\end{array}$
H $\quad-5.729272-1.839586-1.276849$ 

H $\quad-5.514883 \quad-0.090976-1.555999$
H $\quad-4.888078 \quad-0.836203 \quad-0.059632$
$\begin{array}{llll}\text { C } & 0.701564 & -4.764771 & -2.187453\end{array}$
C $0.866379-3.306051 \quad-0.226306$
$\begin{array}{llll}\text { C } & 3.268370 & 1.535337 & -2.907326\end{array}$
H $4.3275891 .427138 \quad-2.625769$
$\begin{array}{llll}\text { C } & 2.385849 & 0.467021 & -2.687053\end{array}$
C $\quad-0.744591 \quad-1.178230 \quad 3.788044$
H $\quad-1.114758 \quad-2.091480 \quad 4.303131$
C $1.377235 \quad 1.9162591 .768730$
H $1.993804 \quad 2.834721 \quad 1.907021$
H $1.119536 \quad 1.5547112 .794306$
$\begin{array}{llll}\text { C } & -3.103861 & 1.560731 & -2.231532\end{array}$
H $\quad-2.183245 \quad 2.162524 \quad-2.397930$
$\begin{array}{llll}\text { C } & 0.786581 & -1.256547 & 3.722038\end{array}$
H $\quad \begin{array}{lll}1.147116 & -2.212360 & 3.299132\end{array}$
H $\quad 1.218510-1.1431314 .738263$
H $\quad 1.211077 \quad-0.455753 \quad 3.084948$
$\begin{array}{llll}\text { C } & -3.938336 & 2.289171 & -1.171485\end{array}$
H $\quad-4.916931 \quad 1.800175 \quad-1.000157$
H $\quad-4.145580 \quad 3.336059 \quad-1.479334$
$\begin{array}{llll}H & -3.404278 & 2.317139 & -0.202622\end{array}$
$\begin{array}{llll}\text { C } & 5.929074 & -0.374533 & 2.139470\end{array}$
H $\quad 6.923145 \quad-0.7365392 .440840$
C $\quad-3.848959 \quad 1.438492 \quad-3.565594$
H $\quad-3.2478720 .935252 \quad-4.349552$
H $\quad-4.110005 \quad 2.446607 \quad-3.952184$
H $\quad-4.800005 \quad 0.877919 \quad-3.460989$ 

C $\quad-1.038875 \quad 3.327974 \quad 2.302251$
H $\quad-0.909007 \quad 2.646763 \quad 3.171680$
C $\quad-3.320454 \quad-1.594683 \quad 2.390603$
$\begin{array}{llll}\text { H } & -3.613488 & -2.080629 & 1.434738\end{array}$
C $-3.631968-2.568565 \quad 3.532448$
H $\quad-4.711732 \quad-2.829306 \quad 3.532530$
H $\quad-3.406254 \quad-2.129049 \quad 4.525432$
H $\quad-3.062932 \quad-3.516222 \quad 3.445160$
C $\quad 0.276316 \quad 3.425134 \quad-0.463240$
H $\quad 0.431593 \quad 2.677812-1.269290$
C $-3.200734-2.704731-1.461832$
H $\quad-3.090155-2.795402-0.363702$
H $-2.219182-2.964685-1.905231$
H $\quad-3.931815 \quad-3.473016-1.790268$
$\begin{array}{llll}\text { C } & 1.597886 & 4.185880 & -0.305167\end{array}$
H $\quad 2.449761 \quad 3.497284 \quad-0.145421$
H $1.574526 \quad 4.923801 \quad 0.521443$
H $1.809913 \quad 4.741418$-1.243048
$\begin{array}{llll}\text { C } & -2.547624 & 3.515672 & 2.111748\end{array}$
H $\quad-2.996031 \quad 3.980849 \quad 3.015092$
H $\quad-3.053410 \quad 2.544269 \quad 1.946423$
H $\quad-2.781440 \quad 4.174137 \quad 1.252042$
C $\quad-0.329223 \quad 4.645968 \quad 2.640426$
H $\quad-0.777269 \quad 5.095526 \quad 3.552301$
H $\quad-0.432505 \quad 5.390886 \quad 1.827071$
$\begin{array}{llll}H & 0.751848 & 4.508133 & 2.837177\end{array}$
C $\quad-0.858572 \quad 4.346487-0.927786$
H $\quad-0.620036 \quad 4.762412 \quad-1.929257$ 


$$
\begin{array}{lrrr}
\text { H } & -1.004120 & 5.205778 & -0.242147 \\
\text { H } & -1.825071 & 3.816318 & -1.008892 \\
\text { C } & -4.120327 & -0.288463 & 2.491309 \\
\text { H } & -5.211938 & -0.491051 & 2.468444 \\
\text { H } & -3.906638 & 0.261271 & 3.429564 \\
\text { H } & -3.876423 & 0.387468 & 1.646378 \\
\text { C } & -1.210876 & 0.050130 & 4.574205 \\
\text { H } & -2.302609 & 0.053440 & 4.759318 \\
\text { H } & -0.705218 & 0.096115 & 5.561273 \\
\text { H } & -0.963722 & 0.983628 & 4.029271 \\
\text { H } & 0.152597 & -5.535598 & -2.751919 \\
\text { U } & 0.698725 & -0.569886 & -0.287226 \\
\text { Fe } & -1.048705 & 0.204553 & 0.353318 \\
\text { H } & 2.765714 & -0.519250 & -2.368394 \\
\text { H } & 3.848392 & -0.265976 & -0.583075 \\
\text { H } & 2.827640 & -2.352255 & -0.014417
\end{array}
$$$$
\text { UFe-2 (Spin 4) }
$$

$$
\begin{array}{lrrr}
P & -2.510012 & -0.534039 & -1.163405 \\
P & -1.352825 & -0.357050 & 2.354879 \\
P & 0.023618 & 2.512071 & 0.241647 \\
N & -0.101037 & -1.390739 & -2.222741 \\
N & 2.213194 & 1.059208 & 0.540079 \\
N & 0.359087 & -2.185096 & 1.613197 \\
C & 4.242993 & -0.224861 & 0.175488 \\
C & -3.994535 & -1.690342 & -0.944549 \\
H & -4.206805 & -2.020954 & -1.986341 \\
C & 2.013322 & -3.084706 & 0.167058 \\
C & -0.018972 & 0.285849 & -4.091516
\end{array}
$$




$$
\begin{array}{lrrr}
\text { H } & -1.086758 & 0.196491 & -4.334370 \\
\mathrm{C} & 2.139549 & 1.371064 & -4.511428 \\
\mathrm{H} & 2.738350 & 2.111897 & -5.061031 \\
\mathrm{C} & -1.511286 & -1.634172 & -2.346571 \\
\mathrm{H} & -1.721663 & -2.676398 & -2.033129 \\
\mathrm{H} & -1.908052 & -1.532756 & -3.381975 \\
\mathrm{C} & 2.415500 & -3.982891 & -0.850213 \\
\mathrm{H} & 3.410110 & -3.859076 & -1.308018 \\
\mathrm{C} & 3.522209 & 0.768756 & 0.902634 \\
\mathrm{C} & 0.556173 & -0.538236 & -3.073446 \\
\mathrm{C} & 4.205264 & 1.388463 & 1.989931 \\
\mathrm{H} & 3.707959 & 2.177367 & 2.571788 \\
\mathrm{C} & 0.767615 & 1.208297 & -4.791184 \\
\mathrm{H} & 0.295318 & 1.826911 & -5.571518 \\
\mathrm{C} & -0.069703 & -4.367199 & 0.440776 \\
\mathrm{H} & -1.016338 & -4.560504 & 0.966414 \\
\mathrm{C} & 1.585877 & -5.033067 & -1.244132 \\
\mathrm{H} & 1.899206 & -5.725646 & -2.038903 \\
\mathrm{C} & -0.881424 & -2.164697 & 2.360332 \\
\mathrm{H} & -0.759834 & -2.558006 & 3.398192 \\
\mathrm{H} & -1.708144 & -2.736184 & 1.884682 \\
\mathrm{C} & 5.529208 & -0.624623 & 0.553434 \\
\mathrm{H} & 6.047482 & -1.398208 & -0.035546 \\
\mathrm{C} & 5.499407 & 0.989258 & 2.352302 \\
\mathrm{H} & 5.991593 & 1.483255 & 3.205666 \\
\mathrm{H} & -5.256262 & -1.021421 & -0.385823 \\
\mathrm{H} & -6.5 .672713 & -0.256797 & -1.067302
\end{array}
$$



H $\quad-5.062671 \quad-0.530567 \quad 0.588030$
$\begin{array}{llll}\text { C } & 0.356026 & -5.232644 & -0.567387\end{array}$
$\begin{array}{llll}\text { C } & 0.727697 & -3.238738 & 0.813121\end{array}$
$\begin{array}{llll}\text { C } & 2.735061 & 0.542422 & -3.546488\end{array}$
H $3.813402 \quad 0.617553 \quad-3.335872$
C $\quad 1.968975-0.413396-2.864669$
C $\quad-0.458458 \quad 0.186582 \quad 3.924920$
H $\quad-0.871864 \quad-0.470414 \quad 4.720701$
C $\quad 1.669024 \quad 2.303864 \quad 1.052462$
$\begin{array}{llll}H & 2.336100 & 3.177163 & 0.860001\end{array}$
H $\quad 1.477674 \quad 2.298053 \quad 2.153946$
C $\quad-3.183153 \quad 0.802660-2.318534$
H $\quad-2.228633 \quad 1.206862 \quad-2.720030$
C $1.052902 \quad-0.069333 \quad 3.843685$
H $1.294955-1.1438853 .751999$
H $\quad 1.553683 \quad 0.324768 \quad 4.752839$
H $\quad \begin{array}{llll}1.511142 & 0.425910 & 2.965079\end{array}$
$\begin{array}{llll}\text { C } & -3.864421 & 1.937931 & -1.543287\end{array}$
H $\quad-4.849743 \quad 1.640947 \quad-1.134839$
H $\quad-4.032519 \quad 2.816336-2.201816$
H $\quad-3.235142 \quad 2.264140 \quad-0.692444$
$\begin{array}{llll}\text { C } & 6.171536 & -0.026833 & 1.652885\end{array}$
H $\quad 7.186393 \quad-0.334231 \quad 1.945615$
C $\quad-4.032652 \quad 0.320729 \quad-3.500124$
H $\quad-3.543975 \quad-0.486367 \quad-4.081872$
H $\quad-4.217578 \quad 1.161434 \quad-4.201735$
H $\quad-5.024775 \quad-0.053735 \quad-3.176253$
C $\quad-0.625426 \quad 4.029305 \quad 1.145872$ 

H $\quad-0.477121 \quad 3.705954 \quad 2.199681$
C $\quad-3.161821 \quad-0.396219 \quad 2.879990$
H $\quad-3.564147 \quad-1.140197 \quad 2.159997$
C $\quad-3.446675 \quad-0.910002 \quad 4.295841$
H $\quad-4.538549 \quad-1.058846 \quad 4.436862$
H $\quad-3.116722 \quad-0.190864 \quad 5.073067$
H $\quad-2.951986-1.880750 \quad 4.500860$
C $\quad 0.493040 \quad 3.005394 \quad-1.521866$
H $0.581482 \quad 2.010995 \quad-2.009430$
$\begin{array}{llll}\text { C } & -3.637542 & -2.935592 & -0.123738\end{array}$
H $\quad-3.556214 \quad-2.692403 \quad 0.954202$
H $\quad-2.681035 \quad-3.404442 \quad-0.426566$
H $\quad-4.433912 \quad-3.701858 \quad-0.226276$
C $\quad 1.846146 \quad 3.703891-1.700850$
H $2.680325 \quad 3.072455 \quad-1.339138$
H $\quad 1.895007 \quad 4.686239-1.191406$
H $\quad 2.020508 \quad 3.876486 \quad-2.783224$
C $\quad-2.131472 \quad 4.2401290 .955529$
H $\quad-2.500461 \quad 5.032191 \quad 1.640776$
H $\quad-2.693966 \quad 3.312013 \quad 1.175439$
H $\quad-2.382102 \quad 4.554504 \quad-0.076819$
$\begin{array}{llll}\text { C } & 0.173147 & 5.324502 & 0.945934\end{array}$
H $\quad-0.189053 \quad 6.104762 \quad 1.648773$
H $\quad 0.053186 \quad 5.729284-0.078361$
H $1.256425 \quad 5.1889381 .132049$
C $\quad-0.641672 \quad 3.747113 \quad-2.239123$
H $\quad-0.440887 \quad 3.770093 \quad-3.330168$
H $\quad-0.736986 \quad 4.796901 \quad-1.895375$ 


$$
\begin{array}{lrrr}
\text { H } & -1.620870 & 3.254817 & -2.091376 \\
\mathrm{C} & -3.863889 & 0.934904 & 2.582658 \\
\mathrm{H} & -4.963240 & 0.839143 & 2.709598 \\
\mathrm{H} & -3.524624 & 1.747583 & 3.255284 \\
\mathrm{H} & -3.661699 & 1.257339 & 1.540726 \\
\mathrm{C} & -0.767142 & 1.641142 & 4.290583 \\
\mathrm{H} & -1.840976 & 1.814021 & 4.498571 \\
\mathrm{H} & -0.199284 & 1.944225 & 5.194882 \\
\mathrm{H} & -0.474876 & 2.326294 & 3.470009 \\
\mathrm{H} & -0.276537 & -6.093647 & -0.837073 \\
\mathrm{U} & 0.727662 & -0.684269 & -0.128098 \\
\mathrm{Fe} & -1.082398 & 0.507538 & 0.293749 \\
\mathrm{H} & 2.463108 & -1.178342 & -2.235121 \\
\mathrm{H} & 3.786111 & -0.652396 & -0.730350 \\
\mathrm{H} & 2.764615 & -2.435938 & 0.654013
\end{array}
$$

\section{UFe-2 (Spin 6)}

$$
\begin{array}{lrrr}
\mathrm{P} & 0.142366 & -2.394485 & -0.995327 \\
\mathrm{P} & 1.749885 & -0.612618 & 2.008206 \\
\mathrm{P} & -2.141258 & -0.270549 & 1.431202 \\
\mathrm{~N} & -0.494351 & -0.081487 & -2.431118 \\
\mathrm{~N} & -1.143488 & 2.119945 & 0.774909 \\
\mathrm{~N} & 2.627617 & 0.582322 & -0.298252 \\
\mathrm{C} & -0.255907 & 4.099942 & -0.300461 \\
\mathrm{C} & 1.610047 & -3.532078 & -1.324989 \\
\mathrm{H} & 1.439724 & -3.910218 & -2.357457 \\
\mathrm{C} & 3.046114 & 1.275294 & -2.585088 \\
\mathrm{C} & -2.510923 & -0.901441 & -3.653640
\end{array}
$$




$$
\begin{array}{lrrr}
\text { H } & -2.105457 & -1.914398 & -3.778969 \\
\text { C } & -4.371319 & 0.647701 & -4.006166 \\
\text { H } & -5.382227 & 0.853871 & -4.387595 \\
\text { C } & 0.226982 & -1.331544 & -2.570503 \\
\text { H } & 1.307218 & -1.125441 & -2.732959 \\
\text { H } & -0.088390 & -1.942752 & -3.443791 \\
\text { C } & 3.912450 & 1.559835 & -3.643389 \\
\text { H } & 3.490798 & 1.862722 & -4.614914 \\
\text { C } & -0.933977 & 3.490732 & 0.799256 \\
\text { C } & -1.740979 & 0.104813 & -3.003624 \\
\mathrm{C} & -1.312612 & 4.341594 & 1.875881 \\
\text { H } & -1.845514 & 3.924582 & 2.742333 \\
\text { C } & -3.799230 & -0.626871 & -4.139356 \\
\text { H } & -4.365358 & -1.434128 & -4.631505 \\
\mathrm{C} & 4.949711 & 0.783331 & -1.156169 \\
\text { H } & 5.383571 & 0.495786 & -0.188172 \\
\mathrm{C} & 5.307786 & 1.457882 & -3.479522 \\
\mathrm{H} & 5.988342 & 1.680361 & -4.314697 \\
\mathrm{C} & 3.153443 & 0.040190 & 0.939636 \\
\mathrm{H} & 3.738785 & 0.782353 & 1.536005 \\
\mathrm{H} & 3.848487 & -0.814855 & 0.752821 \\
\mathrm{C} & 0.061958 & 5.460971 & -0.301942 \\
\mathrm{H} & 0.590309 & 5.889543 & -1.168043 \\
\mathrm{C} & -1.002646 & 5.710033 & 1.855581 \\
\mathrm{H} & -1.310339 & 6.339967 & 2.705807 \\
\mathrm{C} & 1.652248 & -4.726408 & -0.362228 \\
\mathrm{H} & 2.580865 & -5.314682 & -0.518587 \\
\mathrm{H} & 0.799220 & -5.417663 & -0.497963
\end{array}
$$



H $\quad 1.644070 \quad-4.390005 \quad 0.695115$
$\begin{array}{llll}\text { C } & 5.809447 & 1.069228 & -2.228652\end{array}$
$\begin{array}{llll}\text { C } & 3.537001 & 0.875028 & -1.303863\end{array}$
$\begin{array}{llll}\text { C } & -3.612099 & 1.662869 & -3.391503\end{array}$
H $\quad-4.026547 \quad 2.678346 \quad-3.290641$
$\begin{array}{llll}\text { C } & -2.326023 & 1.403946 & -2.912457\end{array}$
C $\quad 1.817464 \quad 0.598057 \quad 3.447065$
$\begin{array}{llll}H & 2.860146 & 0.525895 & 3.825286\end{array}$
$\begin{array}{llll}\text { C } & -1.954884 & 1.538650 & 1.821491\end{array}$
H $\quad-2.951363 \quad 2.029153 \quad 1.924128$
H $\quad-1.478555 \quad 1.590856 \quad 2.833611$

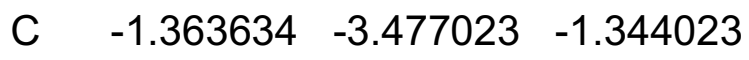
H $\quad-2.130688 \quad-2.697849 \quad-1.545131$
$\begin{array}{llll}\text { C } & 1.582600 & 2.037183 & 2.972614\end{array}$
$\begin{array}{llll}H & 2.272427 & 2.339179 & 2.159320\end{array}$
H $\quad 1.730789 \quad 2.747319 \quad 3.812668$
H $\quad 0.553753 \quad 2.183283 \quad 2.593405$
C $\quad-1.803892 \quad-4.257599-0.097676$
H $\quad-1.116510 \quad-5.0935590 .136206$
H $\quad-2.814989-4.693143 \quad-0.242782$
$\begin{array}{llll}\mathrm{H} & -1.838917 & -3.603112 & 0.797544\end{array}$
$\begin{array}{llll}\text { C } & -0.308121 & 6.283660 & 0.778920\end{array}$
H $\quad-0.068912 \quad 7.357327 \quad 0.772570$
C $\quad-1.263147 \quad-4.388449-2.572072$
H $\quad \begin{array}{llll}-0.917332 & -3.857291 & -3.481559\end{array}$
H $\quad-2.258155 \quad-4.820806 \quad-2.808187$
H $\quad-0.572070 \quad-5.238454 \quad-2.399673$
$\begin{array}{llll}\text { C } & -2.842079 & -0.859326 & 3.086973\end{array}$ 


$$
\begin{array}{lrrr}
\mathrm{H} & -2.068593 & -0.456631 & 3.778582 \\
\mathrm{C} & 2.578064 & -2.181374 & 2.698890 \\
\mathrm{H} & 2.922159 & -2.661112 & 1.757016 \\
\mathrm{C} & 3.802678 & -1.945086 & 3.588277 \\
\mathrm{H} & 4.322145 & -2.905302 & 3.793311 \\
\mathrm{H} & 3.521768 & -1.513986 & 4.571003 \\
\mathrm{H} & 4.541936 & -1.265415 & 3.118274 \\
\mathrm{C} & -3.508741 & -0.310688 & 0.128206 \\
\mathrm{H} & -2.906296 & -0.312784 & -0.803599 \\
\mathrm{C} & 2.945621 & -2.778754 & -1.306667 \\
\mathrm{H} & 3.177430 & -2.407541 & -0.290646 \\
\mathrm{H} & 2.978910 & -1.902613 & -1.981175 \\
\mathrm{H} & 3.767715 & -3.461141 & -1.607038 \\
\mathrm{C} & -4.414311 & 0.924627 & 0.070403 \\
\mathrm{H} & -3.834129 & 1.851198 & -0.103379 \\
\mathrm{H} & -5.023934 & 1.050298 & 0.987924 \\
\mathrm{H} & -5.109836 & 0.819185 & -0.787806 \\
\mathrm{C} & -2.814140 & -2.385037 & 3.233515 \\
\mathrm{H} & -3.049513 & -2.680244 & 4.277698 \\
\mathrm{H} & -1.809366 & -2.790154 & 2.986535 \\
\mathrm{H} & -3.550993 & -2.883970 & 2.573894 \\
\mathrm{C} & -4.191736 & -0.252775 & 3.492606 \\
\mathrm{H} & -4.438843 & -0.536756 & 4.537792 \\
\mathrm{H} & -5.017584 & -0.619052 & 2.851551 \\
\mathrm{H} & -4.191650 & 0.853577 & 3.442856 \\
\mathrm{C} & -4.323668 & -1.610945 & 0.139772 \\
\mathrm{H} & -4.901035 & -1.689789 & -0.804447 \\
\mathrm{H} & -5.050214 & -1.641950 & 0.976621
\end{array}
$$




$$
\begin{array}{lrrr}
\mathrm{H} & -3.686807 & -2.510578 & 0.214228 \\
\mathrm{C} & 1.556658 & -3.128409 & 3.340143 \\
\mathrm{H} & 2.015462 & -4.115729 & 3.558359 \\
\mathrm{H} & 1.152125 & -2.730740 & 4.292252 \\
\mathrm{H} & 0.688985 & -3.300674 & 2.663021 \\
\mathrm{C} & 0.856117 & 0.208044 & 4.573644 \\
\mathrm{H} & 1.111354 & -0.766018 & 5.034118 \\
\mathrm{H} & 0.867661 & 0.971759 & 5.379016 \\
\mathrm{H} & -0.185268 & 0.135377 & 4.197647 \\
\mathrm{H} & 6.897789 & 0.988162 & -2.075846 \\
\mathrm{U} & 0.306337 & 0.738940 & -0.430099 \\
\mathrm{Fe} & -0.161715 & -1.386033 & 1.023582 \\
\mathrm{H} & -1.740283 & 2.218412 & -2.454944 \\
\mathrm{H} & 0.000395 & 3.491648 & -1.189600 \\
\mathrm{H} & 1.955661 & 1.355233 & -2.754743
\end{array}
$$

UCo-2 (Spin 1)
$\begin{array}{crrr}\text { P } & 2.386784 & 0.215541 & -1.433622 \\ \text { P } & 1.365458 & 1.092145 & 2.165665 \\ \text { P } & 0.336434 & -2.301240 & 0.760552 \\ \text { N } & -0.125613 & 0.683413 & -2.517696 \\ \text { N } & -2.011636 & -1.040648 & 0.871507 \\ \text { N } & -0.526804 & 2.492325 & 1.020877 \\ \text { C } & -4.194606 & -0.080193 & 0.401907 \\ \text { C } & 3.711831 & 1.564359 & -1.578937 \\ \text { H } & 3.882883 & 1.654526 & -2.675232 \\ \text { C } & -2.275088 & 2.857482 & -0.538388 \\ \text { C } & -0.347527 & -1.435377 & -3.841465\end{array}$



H $\quad 0.713834 \quad-1.464618 \quad-4.126379$
C $-2.549823 \quad-2.511409-3.859468$
$\begin{array}{llll}\text { H } & -3.193037 & -3.351825 & -4.158321\end{array}$
C $1.267283 \quad 0.861913 \quad-2.825426$
H $1.481513 \quad 1.946007 \quad-2.912008$
H $1.575966 \quad 0.404905-3.790769$
$\begin{array}{llll}\text { C } & -2.777134 & 3.444257 & -1.725914\end{array}$
H $\quad-3.758306 \quad 3.120643 \quad-2.108953$
C $-3.323786-0.807293 \quad 1.266146$
C $-0.854254-0.343088-3.069926$
$\begin{array}{llll}\text { C } & -3.865812 & -1.231049 & 2.514573\end{array}$
$\begin{array}{llll}\text { H } & -3.248851 & -1.816088 & 3.210937\end{array}$
C $-1.190078-2.483144 \quad-4.229363$
H $\quad-0.771440 \quad-3.311178-4.824050$
C $\quad-0.332522 \quad 4.368490-0.643928$
H $\quad 0.597866 \quad 4.767723 \quad-0.214077$
C $\quad-2.065575 \quad 4.449575 \quad-2.379731$
$\begin{array}{llll}\text { H } & -2.457589 & 4.901720 & -3.302488\end{array}$
C $\quad 0.691600 \quad 2.785763 \quad 1.748629$
H $\quad 0.495938 \quad 3.389629 \quad 2.666683$
H $1.458328 \quad 3.329517 \quad 1.154258$
$\begin{array}{llll}\text { C } & -5.494737 & 0.263232 & 0.787648\end{array}$
H $\quad-6.132104 \quad 0.8283050 .088857$
$\begin{array}{llll}\text { C } & -5.174229 & -0.891388 & 2.886274\end{array}$
H $\quad-5.555678 \quad-1.226999 \quad 3.864167$
$\begin{array}{llll}\text { C } & 5.050494 & 1.229552 & -0.910689\end{array}$
H $\quad 5.730667 \quad 2.106838-0.951404$
H $\quad 5.574064 \quad 0.388350-1.402443$ 

H $\quad 4.919443 \quad 0.9626510 .156605$
C $\quad-0.857026 \quad 4.926237 \quad-1.810124$
$\begin{array}{llll}\text { C } & -1.006423 & 3.288548 & 0.007338\end{array}$
C $-3.075056-1.432130-3.131226$
H $\quad-4.142222 \quad-1.411164-2.860071$
C $\quad-2.254786-0.352380-2.774193$
C $\quad 0.550196 \quad 0.880837 \quad 3.858439$
H $\quad 0.917721 \quad 1.751668 \quad 4.444430$
C $-1.289587-2.0586881 .614643$
H $\quad-1.862036 \quad-3.0116991 .690215$
$\begin{array}{llll}\text { H } & -1.044406 & -1.752951 & 2.660537\end{array}$
C $3.217076-1.263029-2.269349$
H $2.318520 \quad-1.870954-2.514729$
$\begin{array}{llll}\text { C } & -0.979711 & 0.985198 & 3.785307\end{array}$
H $\quad-1.319618 \quad 1.964155 \quad 3.400017$
H $\quad-1.416223 \quad 0.839977 \quad 4.795843$
H $\quad-1.422488 \quad 0.219081 \quad 3.119139$
$\begin{array}{llll}\text { C } & 4.061627 & -2.072411 & -1.279294\end{array}$
H $5.027029 \quad-1.579777 \quad-1.052707$
H $4.295932 \quad-3.078674-1.687109$
H $3.523056 \quad-2.209868-0.323146$
$\begin{array}{llll}\text { C } & -5.998761 & -0.132036 & 2.039839\end{array}$
$\begin{array}{llll}\text { H } & -7.023688 & 0.130404 & 2.341115\end{array}$
$\begin{array}{llll}\text { C } & 3.977858 & -0.982897 & -3.570695\end{array}$
H $3.373739-0.423668-4.313205$
H $4.277082 \quad-1.939165-4.050070$
H $4.908483 \quad-0.406551 \quad-3.393544$
$\begin{array}{llll}\text { C } & 1.168714 & -3.433718 & 2.014781\end{array}$ 

H $1.057158 \quad-2.814692 \quad 2.931313$
C $\quad 3.165002 \quad 1.434875 \quad 2.603061$
H $3.483973 \quad 2.026071 \quad 1.718347$
C $3.419219 \quad 2.285978 \quad 3.852015$
H $4.492717 \quad 2.565405 \quad 3.911987$
H $3.176429 \quad 1.738206 \quad 4.785066$
H $\quad 2.8331693 .227291 \quad 3.848722$
C $\quad-0.170264-3.325732 \quad-0.748326$
H $\quad-0.331259-2.518987-1.493576$
$\begin{array}{llll}\text { C } & 3.175531 & 2.907760 & -1.073805\end{array}$
$\begin{array}{llll}H & 3.144617 & 2.926321 & 0.032574\end{array}$
H $\quad 2.149676 \quad 3.131864 \quad-1.427812$
H $3.834901 \quad 3.738545 \quad-1.401194$
C $-1.484765-4.110255-0.659905$
H $\quad-2.342228 \quad-3.444500 \quad-0.442787$
H $\quad-1.455092 \quad-4.917368 \quad 0.098978$
H $\quad-1.689173 \quad-4.583324-1.643387$
C $2.672875-3.619195 \quad 1.791266$
H $3.122084 \quad-4.162365 \quad 2.649528$
H $\quad 3.191247 \quad-2.645051 \quad 1.703761$
H $\quad 2.891633 \quad-4.2078640 .878989$
C $\quad 0.460124 \quad-4.772251 \quad 2.265942$
H $\quad 0.927559 \quad-5.291525 \quad 3.129651$
H $\quad 0.542066 \quad-5.451411 \quad 1.394678$
H $\quad-0.615492 \quad-4.649200 \quad 2.497752$
$\begin{array}{llll}\text { C } & 0.975659 & -4.192962 & -1.284297\end{array}$
H $0.745702 \quad-4.520831-2.319772$
H $\quad \begin{array}{lll}1.124682 & -5.107381 & -0.674910\end{array}$ 


$\begin{array}{cccc}H & 1.937460 & -3.648893 & -1.313400 \\ \mathrm{C} & 3.988783 & 0.140031 & 2.592646 \\ \mathrm{H} & 5.075392 & 0.361573 & 2.653066 \\ \mathrm{H} & 3.739168 & -0.523240 & 3.444616 \\ \mathrm{H} & 3.804236 & -0.431727 & 1.659892 \\ \mathrm{C} & 0.990713 & -0.398490 & 4.576192 \\ \mathrm{H} & 2.088410 & -0.466920 & 4.705042 \\ \mathrm{H} & 0.532919 & -0.450651 & 5.586159 \\ \mathrm{H} & 0.664032 & -1.302025 & 4.022834 \\ \mathrm{H} & -0.320955 & 5.757641 & -2.295077 \\ \mathrm{U} & -0.767385 & 0.597968 & -0.281804 \\ \mathrm{Co} & 1.184491 & -0.249497 & 0.398645 \\ \mathrm{H} & -2.694322 & 0.567852 & -2.346719 \\ \mathrm{H} & -3.843809 & 0.173175 & -0.609825 \\ \mathrm{H} & -2.953138 & 2.284299 & 0.121083 \\ \mathrm{UCo}-2 & (\mathrm{Sp} i n 3) & & \\ \mathrm{P} & -2.357086 & -0.210329 & -1.456134 \\ \mathrm{P} & -1.421974 & -1.107977 & 2.126342 \\ \mathrm{P} & -0.342104 & 2.308659 & 0.768031 \\ \mathrm{~N} & 0.180748 & -0.668836 & -2.507565 \\ \mathrm{~N} & 2.007014 & 1.056582 & 0.901836 \\ \mathrm{~N} & 0.500505 & -2.510842 & 1.013386 \\ \mathrm{C} & 4.178782 & 0.069238 & 0.450407 \\ \mathrm{C} & -3.683046 & -1.554993 & -1.651691 \\ \mathrm{H} & -3.836645 & -1.616479 & -2.752504 \\ \mathrm{C} & 2.302494 & -2.906739 & -0.480898 \\ \mathrm{C} & 0.411973 & 1.462304 & -3.808136 \\ \mathrm{H} & -0.650411 & 1.499315 & -4.087823\end{array}$




$$
\begin{array}{lrrr}
\mathrm{C} & 2.616696 & 2.531728 & -3.815883 \\
\mathrm{H} & 3.261356 & 3.375306 & -4.102513 \\
\mathrm{C} & -1.208999 & -0.844179 & -2.832813 \\
\mathrm{H} & -1.421478 & -1.927325 & -2.932095 \\
\mathrm{H} & -1.505478 & -0.381276 & -3.799187 \\
\mathrm{C} & 2.832687 & -3.485651 & -1.656235 \\
\mathrm{H} & 3.831630 & -3.174700 & -2.001977 \\
\mathrm{C} & 3.311990 & 0.808874 & 1.308289 \\
\mathrm{C} & 0.916284 & 0.357637 & -3.052464 \\
\mathrm{C} & 3.849095 & 1.226117 & 2.560877 \\
\mathrm{H} & 3.234044 & 1.820907 & 3.250633 \\
\mathrm{C} & 1.255801 & 2.513324 & -4.182663 \\
\mathrm{H} & 0.837397 & 3.352066 & -4.762343 \\
\mathrm{C} & 0.336108 & -4.369623 & -0.669884 \\
\mathrm{H} & -0.617668 & -4.752430 & -0.278678 \\
\mathrm{C} & 2.124315 & -4.470745 & -2.347019 \\
\mathrm{H} & 2.537736 & -4.920108 & -3.261643 \\
\mathrm{C} & -0.745616 & -2.797538 & 1.695055 \\
\mathrm{H} & -0.591669 & -3.423624 & 2.606300 \\
\mathrm{H} & -1.498326 & -3.314536 & 1.061201 \\
\mathrm{C} & 5.470842 & -0.292528 & 0.846910 \\
\mathrm{H} & 6.106355 & -0.866337 & 0.153503 \\
\mathrm{C} & 5.150071 & 0.869562 & 2.942113 \\
\mathrm{H} & 5.529600 & 1.201931 & 3.921836 \\
\mathrm{C} & -5.029571 & -1.226425 & -0.995850 \\
\mathrm{H} & -5.714946 & -2.097609 & -1.067416 \\
\mathrm{H} & -5.5 .912095 & -0.986029 & 0.079093
\end{array}
$$



C $\quad 0.889432 \quad-4.926136 \quad-1.823167$
$\begin{array}{llll}\text { C } & 1.007210 & -3.307211 & 0.014588\end{array}$
C $3.140931 \quad 1.438140-3.109090$
H $4.209790 \quad 1.408069-2.845383$
$\begin{array}{llll}\text { C } & 2.318885 & 0.354874 & -2.765916\end{array}$
$\begin{array}{llll}\text { C } & -0.642633 & -0.912859 & 3.834478\end{array}$
H $\quad-0.998684 \quad-1.795254 \quad 4.409680$
$\begin{array}{llll}\text { C } & 1.281200 & 2.079250 & 1.634261\end{array}$
H $\quad 1.852979 \quad 3.033417 \quad 1.704952$
H $\quad 1.033181 \quad 1.784058 \quad 2.682880$
$\begin{array}{llll}\text { C } & -3.180355 & 1.269834 & -2.299165\end{array}$
H $\quad-2.279521 \quad 1.881094 \quad-2.525343$
C $0.889610 \quad-0.978752 \quad 3.775153$
H $\quad 1.259371-1.948695 \quad 3.394691$
H $\quad \begin{array}{lll}1.315359 & -0.818337 & 4.787830\end{array}$
H $\quad 1.312466-0.200954 \quad 3.109363$
$\begin{array}{llll}\text { C } & -4.040447 & 2.071932 & -1.316092\end{array}$
H $\quad-5.004746 \quad 1.572988-1.098836$
H $\quad-4.277268 \quad 3.077644 \quad-1.723879$
H $\quad-3.510979 \quad 2.209630-0.354747$

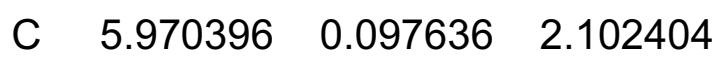
H $\quad 6.989700 \quad-0.177572 \quad 2.411229$
$\begin{array}{llll}\text { C } & -3.919592 & 0.999172 & -3.614688\end{array}$
H $\quad-3.306874 \quad 0.436616 \quad-4.347657$
H $\quad-4.202097 \quad 1.959203 \quad-4.096749$
H $\quad-4.858445 \quad 0.430913 \quad-3.456268$
$\begin{array}{llll}\text { C } & -1.183701 & 3.439704 & 2.021112\end{array}$
$\begin{array}{llll}\mathrm{H} & -1.050990 & 2.832811 & 2.943210\end{array}$ 


$$
\begin{aligned}
& \text { C } \quad-3.228787 \quad-1.464049 \quad 2.515576 \\
& \text { H } \quad-3.528055 \quad-2.025563 \quad 1.604870 \\
& \begin{array}{llll}
\text { C } & -3.500720 & -2.357194 & 3.731007
\end{array} \\
& \text { H } \quad-4.574006 \quad-2.641545 \quad 3.765285 \\
& \text { H } \quad-3.273358 \quad-1.840885 \quad 4.685838 \\
& \text { H } \quad-2.910969-3.295751 \quad 3.705071 \\
& \begin{array}{llll}
\text { C } & 0.167550 & 3.344160 & -0.731465
\end{array} \\
& \text { H } \quad 0.349755 \quad 2.540643 \quad-1.475392 \\
& \text { C } \quad-3.172810 \quad-2.917039-1.171388 \\
& \text { H } \quad-3.120727 \quad-2.948517 \quad-0.065838 \\
& \text { H } \quad-2.163053 \quad-3.170663 \quad-1.550580
\end{aligned}
$$

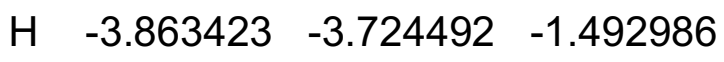

$$
\begin{aligned}
& \text { C } \quad 1.466451 \quad 4.152098-0.627584 \\
& \text { H } \quad 2.335079 \quad 3.503388 \text {-0.403755 } \\
& \text { H } \quad 1.414074 \quad 4.955496 \quad 0.134005 \\
& \text { H } \quad 1.6722864 .633171 \quad-1.606991 \\
& \text { C } \quad-2.692948 \quad 3.591191 \quad 1.808339 \\
& \text { H } \quad-3.148699 \quad 4.130337 \quad 2.665759 \\
& \text { H } \quad-3.188837 \quad 2.604457 \quad 1.731455 \\
& \text { H } \quad-2.931376 \quad 4.168115 \quad 0.893315 \\
& \text { C } \quad-0.499897 \quad 4.793819 \quad 2.255169 \\
& \text { H } \quad-0.963898 \quad 5.305884 \quad 3.125047 \\
& \text { H } \quad-0.611785 \quad 5.468447 \quad 1.383642 \\
& \text { H } \quad 0.581845 \quad 4.694633 \quad 2.469252 \\
& \text { C } \quad-0.988090 \quad 4.192535-1.276208 \\
& \text { H } \quad-0.754882 \quad 4.530519 \quad-2.307673 \\
& \text { H } \quad-1.160941 \quad 5.100274 \quad-0.663401 \\
& \text { H } \quad-1.938896 \quad 3.630514 \quad-1.316250
\end{aligned}
$$




$$
\begin{array}{llll}
\mathrm{C} & -4.056927 & -0.171842 & 2.534201 \\
\mathrm{H} & -5.143638 & -0.399521 & 2.561052 \\
\mathrm{H} & -3.830324 & 0.459187 & 3.416518 \\
\mathrm{H} & -3.854583 & 0.436743 & 1.628814 \\
\mathrm{C} & -1.122498 & 0.356653 & 4.543348 \\
\mathrm{H} & -2.212603 & 0.355003 & 4.737690 \\
\mathrm{H} & -0.609606 & 0.475894 & 5.520478 \\
\mathrm{H} & -0.895846 & 1.255980 & 3.935891 \\
\mathrm{H} & 0.352092 & -5.740371 & -2.335429 \\
\mathrm{U} & 0.784595 & -0.584934 & -0.256616 \\
\mathrm{Co} & -1.194158 & 0.263232 & 0.390737 \\
\mathrm{H} & 2.756555 & -0.572954 & -2.355131 \\
\mathrm{H} & 3.833297 & -0.175344 & -0.565573 \\
\mathrm{H} & 2.958463 & -2.322306 & 0.189249
\end{array}
$$

UCo-2 (Spin 5)

$$
\begin{array}{cccc}
P & -0.636268 & 2.413206 & -0.837835 \\
\text { P } & -1.386231 & 0.470101 & 2.181199 \\
\text { P } & 2.296823 & 0.367422 & 1.258222 \\
\text { N } & 0.055583 & 0.268654 & -2.487898 \\
\text { N } & 1.478792 & -2.062013 & 0.535971 \\
\text { N } & -2.552378 & -0.729975 & 0.023506 \\
\text { C } & 0.634756 & -4.063267 & -0.531075 \\
\text { C } & -2.213710 & 3.437815 & -0.945052 \\
\text { H } & -2.186403 & 3.839850 & -1.982786 \\
\text { C } & -3.301263 & -1.424593 & -2.180327 \\
\text { C } & 1.863723 & 1.386921 & -3.802753 \\
\text { H } & 1.352948 & 2.358695 & -3.809162
\end{array}
$$



C $\quad 3.828027 \quad 0.069263 \quad-4.431383$
H $4.818130 \quad-0.000565 \quad-4.905373$
C $\quad-0.796212 \quad 1.439959-2.458683$
H $\quad-1.860765 \quad 1.125001-2.500888$
$\begin{array}{llll}H & -0.652555 & 2.126281 & -3.322405\end{array}$
C $-4.311182-1.771457-3.081259$
H $\quad-4.039894 \quad-2.026177 \quad-4.118076$
C $1.350830-3.441600 \quad 0.538856$
C $1.2525590 .259330-3.182794$
$\begin{array}{llll}\text { C } & 1.840090 & -4.296967 & 1.568227\end{array}$
H $2.414149 \quad-3.874013 \quad 2.404933$
$\begin{array}{llll}\text { C } & 3.125790 & 1.284413 & -4.409274\end{array}$
H $\quad 3.567301 \quad 2.181577 \quad-4.872547$
C $\quad-4.963895 \quad-1.107684-0.436439$
H $\quad-5.247315 \quad-0.8643290 .597065$
C $\quad-5.661089-1.788763 \quad-2.679630$
H $\quad-6.455050 \quad-2.058786-3.391574$
$\begin{array}{llll}\text { C } & -2.907920 & -0.231656 & 1.337429\end{array}$
H $\quad-3.354200 \quad-1.011408 \quad 2.001509$
H $\quad-3.667812 \quad 0.585348 \quad 1.280475$
C $0.374852-5.437422-0.539800$
$\begin{array}{llll}\text { H } & -0.186748 & -5.873564 & -1.380889\end{array}$
C $1.589683-5.6765721 .537753$
H $\quad \begin{array}{lll}1.983129 & -6.308489 & 2.350371\end{array}$
C $\quad-2.242712 \quad 4.618799 \quad 0.034503$
$\begin{array}{llll}\text { H } & -3.225077 & 5.133337 & -0.019370\end{array}$
H $-1.4645825 .375023-0.180063$
H $\quad-2.101645 \quad 4.2819591 .081480$ 

C $\quad-5.969521-1.453058-1.353091$
$\begin{array}{llll}\text { C } & -3.593620 & -1.083175 & -0.823055\end{array}$
$\begin{array}{llll}\text { C } & 3.222610 & -1.065824 & -3.856808\end{array}$
H $3.736229-2.039696-3.883417$
$\begin{array}{llll}\text { C } & 1.961659 & -0.978464 & -3.262288\end{array}$
$\begin{array}{llll}\text { C } & -1.206848 & -0.766602 & 3.587858\end{array}$
H $\quad-2.196840 \quad-0.740822 \quad 4.093563$
C $2.323117-1.462874 \quad 1.545060$
H $3.368202-1.852968 \quad 1.529144$
H $\quad \begin{array}{lll}1.953261 & -1.619063 & 2.589838\end{array}$
$\begin{array}{llll}\text { C } & 0.737637 & 3.642414 & -1.238716\end{array}$
H $1.538308 \quad 2.947304 \quad-1.574313$
C $\quad-0.986817 \quad-2.189574 \quad 3.059659$
$\begin{array}{llll}\text { H } & -1.774548 & -2.508660 & 2.348072\end{array}$
H $\quad-0.986525 \quad-2.915708 \quad 3.899230$
H $\quad-0.018747 \quad-2.291995 \quad 2.534851$
C $1.245168 \quad 4.363608 \quad 0.018456$
H $\quad 0.513832 \quad 5.099418 \quad 0.405669$
$\begin{array}{llll}H & 2.184327 & 4.914752 & -0.199117\end{array}$
H $1.452357 \quad 3.643896 \quad 0.836933$
$\begin{array}{llll}\text { C } & 0.848428 & -6.261404 & 0.497760\end{array}$
H $\quad 0.656940 \quad-7.344486 \quad 0.484415$
$\begin{array}{llll}\text { C } & 0.445041 & 4.623961 & -2.379620\end{array}$
H $\quad 0.047787 \quad 4.128279-3.287966$
H $1.377261 \quad 5.148382 \quad-2.677170$
$\begin{array}{llll}\text { H } & -0.284563 & 5.402875 & -2.079463\end{array}$
$\begin{array}{llll}\text { C } & 3.192120 & 0.965342 & 2.805087\end{array}$
H $2.587865 \quad 0.460226 \quad 3.591163$ 

C $\quad \begin{array}{llll}-2.123228 & 1.999855 & 3.020795\end{array}$
$\begin{array}{llll}\text { H } & -2.653788 & 2.461274 & 2.160458\end{array}$
C $\quad-3.154875 \quad 1.726143 \quad 4.119930$
H $\quad-3.654588 \quad 2.671294 \quad 4.421467$
H $\quad-2.687728 \quad 1.302993 \quad 5.032508$
H $\quad-3.948490 \quad 1.026192 \quad 3.788548$
$\begin{array}{llll}\text { C } & 3.430990 & 0.598040 & -0.238498\end{array}$
H $2.6988350 .5434390-1.071358$
C $\quad \begin{array}{llll}-3.480776 & 2.584971 & -0.802330\end{array}$
$\begin{array}{llll}H & -3.616055 & 2.243677 & 0.241821\end{array}$
H $\quad-3.490877 \quad 1.681017 \quad-1.440065$
H $\quad-4.372558 \quad 3.190432 \quad-1.065889$
C $4.452455-0.519983-0.477967$
H $3.959241 \quad-1.503120 \quad-0.604070$
H $\quad 5.201940 \quad-0.596569 \quad 0.334881$
H $4.995509-0.310662-1.422759$
C $3.037252 \quad 2.474503 \quad 3.027522$
H $\quad 3.418494 \quad 2.758841 \quad 4.030863$
H $1.9732712 .779022 \quad 2.970232$
H $3.601355 \quad 3.067152 \quad 2.280791$
$\begin{array}{llll}\text { C } & 4.644532 & 0.494917 & 2.960687\end{array}$
H $\quad 5.025577 \quad 0.762531 \quad 3.969245$
$\begin{array}{llll}\text { H } & 5.314880 & 0.976242 & 2.221631\end{array}$
H $4.749949 \quad-0.601743 \quad 2.848066$
C $4.0784951 .987869-0.302240$
H $\quad 4.495514 \quad 2.150775 \quad-1.317331$
H $\quad 4.911342 \quad 2.095623 \quad 0.421593$
H $3.356114 \quad 2.801240-0.106500$ 

C $\quad-1.032245 \quad 2.984261 \quad 3.456502$
H $\quad-1.475911 \quad 3.954081 \quad 3.766079$
H $\quad-0.436662 \quad 2.603563 \quad 4.310352$
H $\quad-0.328330 \quad 3.180112 \quad 2.619387$
C $\quad-0.133752 \quad-0.361052 \quad 4.601546$
H $\quad-0.358953 \quad 0.602451 \quad 5.098750$
H $\quad-0.035063 \quad-1.131528 \quad 5.394371$
H $\quad 0.856028 \quad-0.257918 \quad 4.113521$
H $\quad-7.018465 \quad-1.461890 \quad-1.015301$
U $\quad-0.275962 \quad-0.881867 \quad-0.504640$
Co $0.2269121 .148585 \quad 0.816530$
H $1.488324 \quad-1.889446 \quad-2.857789$
H $\quad 0.309293 \quad-3.457152 \quad-1.399881$
H $\quad-2.254832 \quad-1.403352 \quad-2.539356$

UNi-2 (Spin 2)

$$
\begin{array}{lrrr}
\text { P } & -1.685148 & -0.257444 & -2.205676 \\
\text { P } & -1.797065 & -1.851838 & 1.297310 \\
\text { P } & -1.778445 & 1.987023 & 0.796770 \\
\text { N } & 0.953407 & 0.103336 & -2.234202 \\
N & 0.842405 & 2.011533 & 1.113311 \\
\text { N } & 0.862376 & -2.041034 & 1.159065 \\
\text { C } & 3.223359 & 1.939043 & 0.804324 \\
\text { C } & -1.661170 & -1.886471 & -3.159325 \\
\text { H } & -1.487955 & -1.589390 & -4.216859 \\
C & 3.226414 & -1.653406 & 1.264193 \\
C & 2.525292 & 1.756258 & -3.248209 \\
\text { H } & 1.707442 & 2.342836 & -3.691480
\end{array}
$$



C $\quad 4.911551 \quad 1.489828-2.771958$
H $\quad 5.943332 \quad 1.862011 \quad-2.857341$
$\begin{array}{llll}\text { C } & -0.195732 & 0.571547 & -2.977977\end{array}$
H $\quad-0.109425 \quad 0.340972 \quad-4.067968$

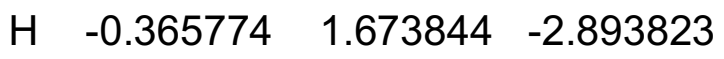
$\begin{array}{llll}\text { C } & 4.544400 & -1.949943 & 0.874992\end{array}$
H $\quad 5.350615 \quad-1.259410 \quad 1.166039$
C $\quad 2.103734 \quad 2.015893 \quad 1.692527$
C $2.228076 \quad 0.552066 \quad-2.543768$
C $2.366228 \quad 1.972272 \quad 3.096278$
H $\quad 1.533130 \quad 2.061175 \quad 3.808634$
$\begin{array}{llll}\text { C } & 3.845147 & 2.208377 & -3.351927\end{array}$
H $\quad 4.050970 \quad 3.146582-3.891633$
$\begin{array}{llll}\text { C } & 2.454456 & -3.726131 & 0.220388\end{array}$
H $\quad \begin{array}{lll}1.649641 & -4.428665 & -0.040675\end{array}$
$\begin{array}{llll}\text { C } & 4.826883 & -3.119416 & 0.155068\end{array}$
H $\quad 5.858297 \quad-3.357563 \quad-0.144382$
C $\quad-0.273715-2.934476 \quad 1.191123$
H $\quad-0.219289-3.651562 \quad 2.046282$
H $\quad-0.384503 \quad-3.5493620 .265635$
$\begin{array}{llll}\text { C } & 4.524527 & 1.743002 & 1.299454\end{array}$
$\begin{array}{llll}\text { H } & 5.359700 & 1.685746 & 0.584574\end{array}$
C $3.670214 \quad 1.792645 \quad 3.568334$
H $3.847384 \quad 1.754290 \quad 4.655089$
C $\quad-2.991565-2.644547 \quad-3.079681$
H $\quad-2.904049 \quad-3.626931 \quad-3.589935$
H $\quad-3.826178-2.097925-3.558357$
H $\quad-3.283366 \quad-2.840418 \quad-2.029753$ 


$$
\begin{array}{lrrr}
\text { C } & 3.773631 & -4.009095 & -0.147388 \\
\text { C } & 2.139717 & -2.516343 & 0.910984 \\
\text { C } & 4.641573 & 0.277947 & -2.119081 \\
\text { H } & 5.459207 & -0.327061 & -1.697886 \\
\text { C } & 3.324614 & -0.200447 & -2.019989 \\
\text { C } & -1.987697 & -1.784334 & 3.171764 \\
\text { H } & -1.906990 & -2.843240 & 3.501186 \\
\text { C } & -0.321200 & 2.384695 & 1.887710 \\
\text { H } & -0.290643 & 3.452006 & 2.208912 \\
\text { H } & -0.463743 & 1.780018 & 2.814715 \\
\text { C } & -3.048960 & 0.735164 & -3.058476 \\
\text { H } & -2.720811 & 1.767832 & -2.811548 \\
\mathrm{C} & -0.837839 & -0.999207 & 3.813347 \\
\text { H } & 0.156383 & -1.380532 & 3.509856 \\
\text { H } & -0.911869 & -1.041205 & 4.920631 \\
\text { H } & -0.880245 & 0.068500 & 3.516292 \\
\mathrm{C} & -4.416463 & 0.526601 & -2.396849 \\
\mathrm{H} & -4.846566 & -0.468100 & -2.628205 \\
\mathrm{H} & -5.143589 & 1.290102 & -2.746229 \\
\mathrm{H} & -4.333727 & 0.602912 & -1.293327 \\
\mathrm{C} & 4.757549 & 1.653946 & 2.679361 \\
\mathrm{H} & 5.776715 & 1.508517 & 3.067278 \\
\mathrm{C} & -3.113738 & 0.623460 & -4.587053 \\
\mathrm{H} & -2.134312 & 0.812320 & -5.069757 \\
\mathrm{H} & -3.829707 & 1.368751 & -4.994899 \\
\mathrm{H} & -3.466234 & -0.374305 & -4.917923 \\
\mathrm{H} & -3.171151 & 2.328626 & 2.025176 \\
& -2.847872 & 1.652840 & 2.849101
\end{array}
$$




$$
\begin{array}{lrrr}
\text { C } & -3.109421 & -3.101942 & 0.765855 \\
\text { H } & -2.674795 & -3.437064 & -0.201960 \\
\text { C } & -3.270675 & -4.336738 & 1.660464 \\
\text { H } & -3.941465 & -5.077926 & 1.175685 \\
\text { H } & -3.726836 & -4.083060 & 2.639036 \\
\text { H } & -2.306941 & -4.846901 & 1.859338 \\
\text { C } & -1.733449 & 3.406029 & -0.459115 \\
\text { H } & -1.169925 & 2.923020 & -1.286112 \\
\mathrm{C} & -0.492280 & -2.774986 & -2.716237 \\
\mathrm{H} & -0.605554 & -3.069849 & -1.653009 \\
\mathrm{H} & 0.483329 & -2.261451 & -2.809795 \\
\mathrm{H} & -0.465333 & -3.706046 & -3.321142 \\
\mathrm{C} & -0.951007 & 4.660095 & -0.051550 \\
\mathrm{H} & 0.105427 & 4.424901 & 0.182573 \\
\mathrm{H} & -1.400342 & 5.170620 & 0.823758 \\
\mathrm{H} & -0.951440 & 5.389386 & -0.889990 \\
\mathrm{C} & -4.526501 & 1.809269 & 1.529809 \\
\mathrm{H} & -5.260656 & 1.774777 & 2.362468 \\
\mathrm{H} & -4.426871 & 0.785091 & 1.114226 \\
\mathrm{H} & -4.955667 & 2.454283 & 0.737976 \\
\mathrm{C} & -3.260788 & 3.747984 & 2.601386 \\
\mathrm{H} & -4.004357 & 3.779923 & 3.426570 \\
\mathrm{H} & -3.590784 & 4.484006 & 1.841712 \\
\mathrm{H} & -2.295933 & 4.100877 & 3.015482 \\
\mathrm{H} & -3.129343 & 3.763937 & -0.989764 \\
\mathrm{H} & -3.044452 & 4.373399 & -1.913993 \\
-3.702954 & 4.366163 & -0.256984 \\
\hline
\end{array}
$$




$$
\begin{array}{lrrr}
\mathrm{C} & -4.449073 & -2.425391 & 0.449682 \\
\mathrm{H} & -5.121461 & -3.118322 & -0.099253 \\
\mathrm{H} & -4.982141 & -2.106417 & 1.367507 \\
\mathrm{H} & -4.293737 & -1.522082 & -0.175163 \\
\mathrm{C} & -3.350185 & -1.237786 & 3.611165 \\
\mathrm{H} & -4.188865 & -1.898312 & 3.319028 \\
\mathrm{H} & -3.384990 & -1.126623 & 4.715557 \\
\mathrm{H} & -3.544408 & -0.241271 & 3.168151 \\
\mathrm{H} & 3.990866 & -4.944554 & -0.687504 \\
\mathrm{U} & 1.009449 & -0.026831 & 0.052081 \\
\mathrm{Ni} & -1.633332 & -0.074156 & -0.000441 \\
\mathrm{H} & 3.142464 & -1.207337 & -1.607670 \\
\mathrm{H} & 3.069731 & 2.128826 & -0.270408 \\
\mathrm{H} & 3.040958 & -0.800657 & 1.940862
\end{array}
$$

UNi-2 (Spin 4)

$$
\begin{array}{lrrr}
P & -1.220364 & -0.973274 & -1.973059 \\
P & 1.459252 & -2.445436 & 0.286865 \\
P & -1.670860 & -0.705916 & 1.901114 \\
N & -0.860354 & 1.719031 & -1.360867 \\
N & -0.012879 & 1.285425 & 2.250743 \\
N & 2.681487 & -0.240008 & -0.648880 \\
C & 1.510847 & 3.048647 & 1.741111 \\
C & -0.730126 & -1.889391 & -3.551262 \\
H & -1.252976 & -1.334403 & -4.360691 \\
C & 2.996722 & 1.978529 & -1.491851 \\
C & -2.879657 & 2.769267 & -2.381481 \\
H & -2.765926 & 2.217655 & -3.324364
\end{array}
$$



C $\quad-4.148271 \quad 4.408344 \quad-1.083778$
H $\quad-4.996529 \quad 5.102399 \quad-0.989931$
C $\quad-0.691497 \quad 0.783487 \quad-2.455491$
H $\quad 0.392713 \quad 0.689669 \quad-2.707144$
H $\quad-1.166368 \quad 1.085693 \quad-3.413889$
C $3.219749 \quad 2.881386-2.543655$
H $3.107335 \quad 3.960969-2.356191$
C $\quad 1.092168 \quad 2.005708 \quad 2.644835$
C $\quad-1.941544 \quad 2.586061 \quad-1.326919$
C $\quad 1.913701 \quad 1.750697 \quad 3.784373$
H $\quad 1.595954 \quad 0.999308 \quad 4.522082$
C $\quad-3.956602 \quad 3.659667-2.254862$
$\begin{array}{llll}\text { H } & -4.658617 & 3.767852 & -3.097451\end{array}$
C $3.591321 \quad 0.121281-2.956919$
H $\quad 3.771227 \quad-0.948406-3.135455$
$\begin{array}{llll}\text { C } & 3.623080 & 2.420947 & -3.806143\end{array}$
H $3.814136 \quad 3.127761 \quad-4.626824$
C $2.837158-1.675202-0.719443$
H $\quad 3.829856-2.021831-0.341580$
H $\quad 2.735396 \quad-2.080290 \quad-1.750957$
C $\quad 2.723185 \quad 3.742499 \quad 1.953877$
H $3.006385 \quad 4.551645 \quad 1.262107$
$\begin{array}{llll}\text { C } & 3.104517 & 2.456591 & 3.974702\end{array}$
H $\quad 3.716398 \quad 2.245666 \quad 4.866238$
C $-1.211208-3.346743 \quad-3.568269$
H $\quad-0.829809 \quad-3.865647 \quad-4.473058$
H $\quad-2.313482-3.434245-3.581666$
$\begin{array}{llll}\text { H } & -0.843017 & -3.905399 & -2.683952\end{array}$ 

C $\quad 3.831822 \quad 1.038852 \quad-3.986623$
$\begin{array}{llll}\text { C } & 3.120421 & 0.560438 & -1.681847\end{array}$
$\begin{array}{llll}\text { C } & -3.222604 & 4.252543 & -0.034197\end{array}$
H $\quad-3.341836 \quad 4.827967 \quad 0.897829$
$\begin{array}{llll}\text { C } & -2.147928 & 3.367693 & -0.150263\end{array}$
C $\quad 2.381617 \quad-2.765855 \quad 1.892806$
H $3.259959-3.383720 \quad 1.605230$
$\begin{array}{llll}\text { C } & -0.573225 & 0.238190 & 3.072697\end{array}$
H $\quad-1.128856 \quad 0.626762 \quad 3.957564$
H $\quad 0.180099 \quad-0.489147 \quad 3.458847$
C $\quad-3.095063 \quad-0.906326 \quad-2.106564$
H $\quad-3.317082-0.041163 \quad-1.443321$
$\begin{array}{llll}\text { C } & 2.897073 & -1.448919 & 2.482808\end{array}$
H $3.553475 \quad-0.891148 \quad 1.787486$
H $3.466193 \quad-1.637785 \quad 3.417119$
H $\quad 2.061811 \quad-0.769383 \quad 2.743400$
C $\quad-3.736879-2.160427 \quad-1.494445$
H $\quad-3.638529-3.041059-2.158480$
H $\quad-4.822517 \quad-2.004590 \quad-1.323405$
H $\quad-3.265944 \quad-2.421762 \quad-0.526189$
C $\quad 3.534697 \quad 3.437363 \quad 3.050877$
H $4.477026 \quad 3.980101 \quad 3.215407$
C $\quad-3.670843 \quad-0.614066 \quad-3.497012$
H $\quad-3.182675 \quad 0.244667 \quad-3.996774$
H $\quad-4.750191 \quad-0.367482-3.416166$
H $\quad-3.586847 \quad-1.491528 \quad-4.170340$
$\begin{array}{llll}\text { C } & -2.239220 & -2.106059 & 3.024591\end{array}$
H $\quad-1.252617 \quad-2.500730 \quad 3.354582$ 


$$
\begin{array}{lrrr}
\mathrm{C} & 1.316726 & -4.149816 & -0.508574 \\
\mathrm{H} & 1.236772 & -3.871069 & -1.581851 \\
\mathrm{C} & 2.533037 & -5.068017 & -0.346838 \\
\mathrm{H} & 2.418976 & -5.971846 & -0.982381 \\
\mathrm{H} & 2.649727 & -5.421171 & 0.698074 \\
\mathrm{H} & 3.478780 & -4.572080 & -0.644690 \\
\mathrm{C} & -3.101022 & 0.509735 & 1.660745 \\
\mathrm{H} & -2.767017 & 1.035236 & 0.741759 \\
\mathrm{C} & 0.773061 & -1.820005 & -3.830687 \\
\mathrm{H} & 1.344278 & -2.364543 & -3.053900 \\
\mathrm{H} & 1.176206 & -0.790672 & -3.869396 \\
\mathrm{H} & 1.002032 & -2.301009 & -4.804655 \\
\mathrm{C} & -3.278431 & 1.576015 & 2.748172 \\
\mathrm{H} & -2.362614 & 2.182193 & 2.885340 \\
\mathrm{H} & -3.568493 & 1.140864 & 3.725666 \\
\mathrm{H} & -4.085984 & 2.273031 & 2.440345 \\
\mathrm{C} & -2.932998 & -3.234738 & 2.251928 \\
\mathrm{H} & -3.072335 & -4.123560 & 2.902968 \\
\mathrm{H} & -2.330242 & -3.547968 & 1.375901 \\
\mathrm{H} & -3.934392 & -2.934325 & 1.885603 \\
\mathrm{C} & -3.018338 & -1.692764 & 4.280385 \\
\mathrm{H} & -3.161772 & -2.571361 & 4.945094 \\
\mathrm{H} & -4.025754 & -1.304660 & 4.032574 \\
\mathrm{H} & -2.493509 & -0.915801 & 4.869920 \\
\mathrm{C} & -4.438446 & -0.170113 & 1.339906 \\
\mathrm{H} & -5.148407 & 0.580975 & 0.935599 \\
\mathrm{H} & -4.907284 & -0.610512 & 2.243015 \\
& -440783 & -0.971350 & 0.588435
\end{array}
$$




$$
\begin{array}{lrrr}
\mathrm{C} & 0.000006 & -4.838461 & -0.125437 \\
\mathrm{H} & -0.158398 & -5.753067 & -0.735022 \\
\mathrm{H} & -0.021120 & -5.141762 & 0.940355 \\
\mathrm{H} & -0.863188 & -4.160582 & -0.295415 \\
\mathrm{C} & 1.535461 & -3.544197 & 2.903640 \\
\mathrm{H} & 1.272502 & -4.560093 & 2.549640 \\
\mathrm{H} & 2.077924 & -3.655800 & 3.865653 \\
\mathrm{H} & 0.585938 & -3.010371 & 3.113854 \\
\mathrm{H} & 4.197125 & 0.666358 & -4.957426 \\
\mathrm{U} & 0.751670 & 0.908028 & 0.090414 \\
\mathrm{Ni} & -0.489630 & -1.399766 & 0.109628 \\
\mathrm{H} & -1.451367 & 3.241931 & 0.694207 \\
\mathrm{H} & 0.775059 & 3.454616 & 1.018772 \\
\mathrm{H} & 2.866166 & 2.376116 & -0.465929
\end{array}
$$

